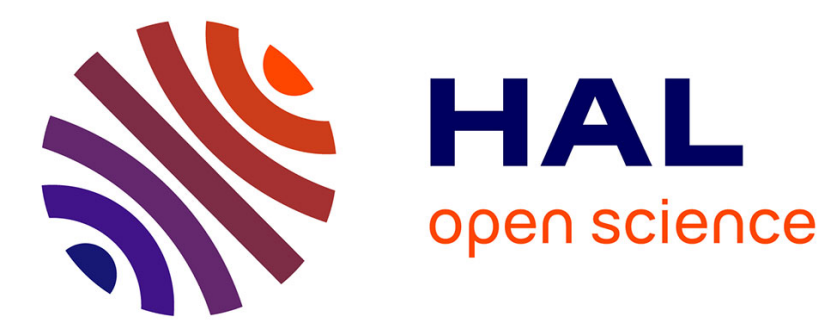

\title{
EXACT OPTIMIZATION VIA SUMS OF NONNEGATIVE CIRCUITS AND SUMS OF AM/GM EXPONENTIALS
}

Victor Magron, Henning Seidler, Timo de Wolff

\section{- To cite this version:}

Victor Magron, Henning Seidler, Timo de Wolff. EXACT OPTIMIZATION VIA SUMS OF NONNEGATIVE CIRCUITS AND SUMS OF AM/GM EXPONENTIALS. ACM Communications in Computer Algebra, 2019, 10.1145/3326229.3326271 . hal-02006899

\section{HAL Id: hal-02006899 \\ https://hal.laas.fr/hal-02006899}

Submitted on 4 Feb 2019

HAL is a multi-disciplinary open access archive for the deposit and dissemination of scientific research documents, whether they are published or not. The documents may come from teaching and research institutions in France or abroad, or from public or private research centers.
L'archive ouverte pluridisciplinaire HAL, est destinée au dépôt et à la diffusion de documents scientifiques de niveau recherche, publiés ou non, émanant des établissements d'enseignement et de recherche français ou étrangers, des laboratoires publics ou privés. 


\title{
EXACT OPTIMIZATION VIA SUMS OF NONNEGATIVE CIRCUITS AND SUMS OF AM/GM EXPONENTIALS
}

\author{
VICTOR MAGRON, HENNING SEIDLER, AND TIMO DE WOLFF
}

\begin{abstract}
We provide two hybrid numeric-symbolic optimization algorithms, computing exact sums of nonnegative circuits (SONC) and sums of arithmetic-geometricexponentials (SAGE) decompositions. Moreover, we provide a hybrid numeric-symbolic decision algorithm for polynomials lying in the interior of the SAGE cone. Each framework, inspired by previous contributions of Parrilo and Peyrl, is a rounding-projection procedure.

For a polynomial lying in the interior of the SAGE cone, we prove that the decision algorithm terminates within a number of arithmetic operations, which is polynomial in the degree and number of terms of the input, and singly exponential in the number of variables. We also provide experimental comparisons regarding the implementation of the two optimization algorithms.
\end{abstract}

\section{INTRODUCTION}

In this paper, we focus on certifying the output of polynomial optimization problems in a rigorous way. Finding the minimal value of a given polynomial in $n$ variables under polynomial constraints is known to be NP-hard in general [Lau09]. The related problem of deciding nonnegativity of a polynomial under polynomial constraints is co-NP hard; see e.g., BCSS12. This decision problem can be solved with the Cylindrical Algebraic Decomposition algorithm [Col75, which runs in time doubly exponential in $n$ and polynomial in the maximal total degree $d$ of the input functions. Further improved algorithms GV88, BPR98, BGHP05, relying on critical point methods, allow to decide nonnegativity in singly exponential time in $n$. More generally, the complement of the problem lies in the existential theory of the reals, which can be solved in polynomial space and single exponential time Ren88. Safe validation of optimization problem results is mandatory for guaranted evaluation of mathematical functions CHJL11, certified roundoff error bounds [MCD17 or computer assisted proofs [MAGW15, $\mathrm{HAB}^{+} 17$ ].

In contrast to the algorithms mentioned above, several numerical frameworks have been developed in the last two decades. In the unconstrained case, one way to ensure nonnegativity of a given polynomial $f$ is to decompose $f$ as a sum of squares (SOS) of polynomials [Par00, Las01], which provides a certificate that $f$ is nonnegative over the

Date: February 4, 2019.

2010 Mathematics Subject Classification. Primary: 14P10,68W30, 90C25; Secondary: 14Q20, 68R01 ACM Subject Classification: Mathematical software performance.

Key words and phrases. nonnegative circuit polynomial, arithmetic-geometric-mean exponential, convex optimization, geometric programming, relative entropy programming, exact certificate, roundingprojection procedure, hybrid numeric-symbolic algorithm, real algebraic geometry. 
reals. An SOS decomposition can be computed by solving a semidefinite program (SDP) of size $\left(\begin{array}{c}n+d \\ n\end{array}\right)$. In the constrained case, certificates can be provided by the prominent momentSOS hierarchy, also called Lasserre's hierarchy [Las01, Las10]. Each relaxation is solved with a semidefinite programming solver, implemented in finite-precision arithmetic, whose output is an approximate certificate. A drawback of these methods is that the size $\left(\begin{array}{c}n+d \\ n\end{array}\right)$ of the SDP matrices blows up when the degree $d$ and number of variables $n$ increases.

For larger values of $n$, a remedy consists of exploiting a potential sparsity/symmetry pattern arising in the input polynomials. A sparse version of Lasserre's hierarchy has been developed in [WKKM06, Las06] when the objective function can be written as a sum of polynomials, each of them involving a small number of variables. The framework from [RTAL13] allows to take into account the symmetries of the polynomial optimization problem. One can also rely on the so-called bounded degree SOS hierarchy (BSOS) [LTY17]. In this hierarchy, positive polynomials are represented as a sum of two terms. The first term is an SOS polynomial of degree fixed in advance, while the second one belongs to the set of Krivine-Stengle representations, that is, is a finite combination of positive scalar weights and cross-products of the polynomials defining the set of constraints. This allows to handle larger instances than with the standard SOS hierarchy. The sparse variant of the BSOS hierarchy [WLT18 can handle even bigger problems, under the same sparsity pattern assumptions than the ones used for the sparse SOS hierarchy. If the support, i.e., number of monomial terms, of the polynomials is small in comparison to $\left(\begin{array}{c}n+d \\ n\end{array}\right)$, alternative relaxations based on geometric programming (GP) [DPZ67], and, more generally, relative entropy programming (REP), potentially allow to obtain lower bounds in a more efficient way than SDP relaxations. Both GP and REP are (equivalent to) convex optimization problems over the exponential cone. These alternative relaxations also provide the possibility to obtain answers when the SDP relaxations cannot be implemented because their size is too large for state-of-the art SDP solvers.

A first class of alternative certificates is given by sums of nonnegative circuit (SONC) polynomials. A circuit polynomial is a polynomial with support containing only monomial squares, at the exception of at most one term, whose exponent is a strict convex combination of the other exponents. In [IdW16], the authors derive a necessary and sufficient condition to prove that a given circuit polynomial is nonnegative. When the input polynomial has a more general support, a first attempt is given in [GM12, GM13] to compute lower bounds while relying on GP. This approach is generalized in [DIdW16] to compute SONC certificates when the set of constraints is defined as a finite conjunction of polynomial inequalities. In [DIdW17] the authors provide a bounded degree hierarchy, which can be computed via relative entropy programming. In the recent contribution [SdW18a], the second and the third author develop an algorithm computing SONC certificates for sparse unconstrained polynomials with arbitrary support, together with a software library [SdW18b], called POEM (Effective Methods in Polynomial Optimization). Although this framework yields a very efficient way to obtain a lower bound for a given polynomial, a drawback is that it currently remains unclear whether the number of 
circuits involved in a SONC relaxation is exponential in the number of terms of this polynomial. A second class of alternative certificates is given by sums of arithmetic-geometricmean-exponentials (SAGE) polynomials. An AGE polynomial refers to a signomial, i.e., a weighted sum of exponentials composed with linear functionals of the variables, which is globally nonnegative with at most one negative coefficient. The framework from CS16 derives a hierarchy of convex relaxations providing a sequence of increasing lower bounds for the optimal value of signomial programs (SP). For an input polynomial belonging to the SAGE cone, one can compute a SAGE decomposition by solving an REP, involving linear and relative entropy functions. Furthermore, it is shown in [MCW18, Theorem 20] that the cones of SAGE and SONC polynomials are related through their equivalence in terms of extreme rays. Namely, the extreme rays of the SAGE cone are supported on either a single coordinate or a set of coordinates inducing a simplicial circuit (a circuit with $\ell$ elements containing $\ell-1$ extreme points). Hence, both cones contain the same polynomials.

However, these alternative schemes share the same certification issues than the ones based on SDP relaxations. GP/REP/SP solvers rely on interior-point algorithms, implemented in finite-precision. Thus, they output only approximate certificates. In the unconstrained case, the rounding-projection procedure by Peryl and Parrilo [PP08] allows to compute a weighted rational SOS decompositon of a polynomial $f$ of degree $d=2 k$, belonging to the interior of the SOS cone. In the "rounding" step, the algorithm computes an approximate Gram matrix of $f$, i.e., a matrix $\widetilde{\boldsymbol{G}}$ such that $f \simeq \boldsymbol{v}_{k}^{T} \widetilde{\boldsymbol{G}} \boldsymbol{v}_{k}$, where $\boldsymbol{v}_{k}$ is the vector of all monomials of degree at most $k$, then rounds $\widetilde{\boldsymbol{G}}$ in the space of rational matrices. In the "projection" step, the algorithm performs an orthogonal projection to obtain a matrix $\boldsymbol{G}$, such that $f=\boldsymbol{v}_{k}^{T} \boldsymbol{G} \boldsymbol{v}_{k}$. With sufficient precision digits, there always exists an SDP matrix fulfilling the above equality, yielding an (exact) Gram matrix associated to $f$. The last step to retrive an exact weighted SOS decomposition for $f$ consists of performing an exact $\boldsymbol{L} \boldsymbol{D} \boldsymbol{L}^{T}$ procedure [GL96, $\S 4.1$ ]. Another framework [MD18a, MSED18], provides a hybrid numeric-symbolic framework, which computes exact SOS decompositions under the same assumptions. This is based on a "perturbation-compensation" algorithm. In the "perturbation" step, one considers an arbitrary small perturbation of the input polynomial, and computes an approximate SOS decomposition with an SDP solver. In the "compensation" step, one relies on the perturbation terms to recover an exact SOS decomposition. By comparison with the rounding-projection procedure, this algorithm perturbates the input and provides an approximate $\boldsymbol{L} \boldsymbol{D} \boldsymbol{L}^{T}$ decomposition of the approximate Gram matrix, instead of a projection. It is shown in MSED18 that both procedures have a boolean running time, which is singly exponential in $n$ and polynomial in $d$. Practical experiments emphasize that the bit size of the SOS outputs obtained with the rounding-projection algorithm is often larger than the one obtained with the perturbationcompensation algorithm. The perturbation-compensation algorithm is inspired from prior work [MDS18], focusing on weighted SOS decompositions for nonnegative univariate polynomials, where the algorithm from [CHJL11] is formalized and analyzed. In the unconstrained case, the framework from [MSED18] also extends the perturbation-compensation 
and rounding-projection algorithms to compute exact Polya and Hilbert-Artin's representations, respectively for positive definite forms and nonnegative polynomials, yielding decompositions into SOS of rational functions, under the assumption that the numerator belongs to the interior of the SOS cone. In the constrained case, further algorithms are proposed to derive exact rational Putinar's representations for positive polynomials over basic compact semialgebraic sets. All corresponding algorithms are integrated in the RealCertify [MD18b] Maple library.

The motivation of the present work is to improve the scalability of these existing certification frameworks, especially for large-size problems, which are currently out of reach when relying on SOS-based methods.

Contributions. In this paper, we provide a hybrid numeric-symbolic framework, in a similar spirit as [PP08, to certify exactly lower bounds obtained after computing SON$\mathrm{C} / \mathrm{SAGE}$ decompositions with $\mathrm{GP} / \mathrm{REP}$ relaxations. The resulting rounding-projection algorithms allow to handle unconstrained polynomial problems with such exact rational decompositions. Our first contributions, given in Section 3, are two procedures, called optsonc and optsage, providing exact rational SONC and SAGE decompositions, respectively. These two algorithms allow to certify exactly lower bounds of unconstrained polynomials. Our framework is inspired from [PP08], first by rounding the output of a given GP/REP relaxation into rational numbers, next by performing an appropriate scaling of these numbers to obtain a solution satisfying exactly the (in)-equality constraints of the relaxation. We present another rounding-projection procedure called intsage in Section 4 to handle the case of polynomials belonging to the interior of the SAGE cone. When the input is an $n$-variate polynomial of degree $d$ with $t$ monomial terms, and integer coefficients of maximum bit size $\tau$, we prove that Algorithm intsage outputs SAGE decompositions within $\mathcal{O}\left(\tau \cdot(4 d+6)^{3 n+3} t^{7} \log t\right)$ arithmetic operations. This is in contrast with the decision algorithm intsos from [MSED18], which certifies nonnegativity of polynomials lying in the interior of the SOS cone in boolean time $\mathcal{O}\left(\tau^{2} \cdot(4 d+2)^{15 n+6}\right)$. The two optimization algorithms optsonc and optsage are available within the POEM software library. In Section 5, we provide experimental comparisons of these two algorithms.

Acknowledgements. Victor Magron benefited from the support of the FMJH Program PGMO (EPICS project) and EDF, Thales, Orange et Criteo. Timo de Wolff and Henning Seidler are supported by the DFG grant WO 2206/1-1. The authors would like to specially acknowledge the help of Riley Murray for providing insights about the barrier complexity of relative entropy programming.

\section{PRELIMINARIES}

Let $\mathbb{Z}$ be the set of integers and let $\mathbb{R}, \mathbb{R}_{\geq 0}$ and $\mathbb{R}_{>0}$ be the set of real, nonnegative real and positive real numbers, respectively. With $\mathbb{Q}$ being the set of rational numbers, one defines similarly $\mathbb{Q}_{>0}, \mathbb{Q}_{\geq 0}$. The bit size of $i \in \mathbb{Z}$ is denoted by $\tau(i):=\left\lfloor\log _{2}(|i|)\right\rfloor+1$ with $\tau(0):=1$. Given $i \in \mathbb{Z}$ and $j \in \mathbb{Z} \backslash\{0\}$ with $\operatorname{gcd}(i, j)=1$, we define $\tau(i / j):=$ $\max \{\tau(i), \tau(j)\}$. For two mappings $g, h: \mathbb{N}^{l} \rightarrow \mathbb{R}$, we use the notation $g(\boldsymbol{v}) \in \mathcal{O}(h(\boldsymbol{v}))$ to state the existence of $i \in \mathbb{N}$ such that $g(\boldsymbol{v}) \leq i h(\boldsymbol{v})$, for all $\boldsymbol{v} \in \mathbb{N}^{l}$. Throughout the paper, 
we use bold letters for vectors (small) and matrices (capital), e.g., $\boldsymbol{x}=\left(x_{1}, \ldots, x_{n}\right) \in \mathbb{R}^{n}$. For a given vector $\boldsymbol{x}$, we denote the $j$-th coordinate of $\boldsymbol{x}$ by $x_{j} \in \mathbb{R}$, and $\boldsymbol{x}_{\backslash j} \in \mathbb{R}^{n-1}$ as the vector obtained by $\boldsymbol{x}$ after removing $x_{j}$. Furthermore, let $\mathbb{R}[\boldsymbol{x}]=\mathbb{R}\left[x_{1}, \ldots, x_{n}\right]$ be the ring of real $n$-variate polynomials. We define $\mathbb{Q}[\boldsymbol{x}]$ similarly. We denote the set of all $n$-variate polynomials of degree less than or equal to $2 d$ by $\mathbb{R}[\boldsymbol{x}]_{n, 2 d}$.

We mostly regard sparse polynomials $p \in \mathbb{R}[\boldsymbol{x}]$ supported on a finite set $A \subset \mathbb{N}^{n}$; we write $A(p)$ if a clarification is necessary. Thus, $p$ is of the form $p(\boldsymbol{x})=\sum_{\boldsymbol{\alpha} \in A} b_{\boldsymbol{\alpha}} \boldsymbol{x}^{\boldsymbol{\alpha}}$ with $b_{\alpha} \in \mathbb{R} \backslash\{0\}$ and $x^{\alpha}=x_{1}^{\alpha_{1}} \cdots x_{n}^{\alpha_{n}}$. Unless stated differently, we follow the convention $t=\# A$. Sparsity then means $t \ll\left(\begin{array}{c}n+2 d \\ 2 d\end{array}\right)=\operatorname{dim}\left(\mathbb{R}[\boldsymbol{x}]_{n, 2 d}\right)$. The support of $p$ can be expressed as an $n \times t$ matrix, which we denote by $\boldsymbol{A}$, such that the $j$-th column of $\boldsymbol{A}$ is $\boldsymbol{\alpha}(\boldsymbol{j})$. Hence, $p$ is uniquely described by the pair $(\boldsymbol{A}, \boldsymbol{b})$, written $p=\operatorname{poly}(\boldsymbol{A}, \boldsymbol{b})$. If $\mathbf{0} \in A$, then $p(\mathbf{0})$ is called the constant term.

Let us denote by $\operatorname{New}(p):=\operatorname{conv}\left(\left\{\boldsymbol{\alpha} \in \mathbb{N}^{n}: b_{\boldsymbol{\alpha}} \neq 0\right\}\right)$ the Newton polytope of $p$ and $\operatorname{Vert}(p):=\{\boldsymbol{\alpha} \in A(p): \boldsymbol{\alpha}$ is vertex of New $(p)\}$ be its vertices. We define $\operatorname{MoSq}(p):=$ $\left\{\boldsymbol{\alpha} \in A(p): \boldsymbol{\alpha} \in(2 \mathbb{N})^{n}, b_{\boldsymbol{\alpha}}>0\right\}$ as the set of monomial squares in the support of $p$. Moreover, we use the notation $\operatorname{NoSq}(p)=A(p) \backslash \operatorname{MoSq}(p)$ for all elements of the support of $p$, which are not monomial squares. We indicate the elements of the support which are in the interior of $\operatorname{New}(p)$ by int $(p)=A(p) \backslash \partial \operatorname{New}(p)$.

2.1. SONC Polynomials. We now introduce the fundamental facts of SONC polynomials, which we use in this article. SONC are constructed by circuit polynomials, which were first introduced in [IdW16]:

Definition 2.1. A circuit polynomial $p=\operatorname{poly}(\boldsymbol{A}, \boldsymbol{b}) \in \mathbb{R}[\boldsymbol{x}]$ is of the form $p(\boldsymbol{x})=$ $\sum_{j=1}^{r} b_{\boldsymbol{\alpha}(j)} \boldsymbol{x}^{\boldsymbol{\alpha}(j)}+b_{\boldsymbol{\beta}} \boldsymbol{x}^{\boldsymbol{\beta}}$, with $0 \leq r<n$, coefficients $b_{\boldsymbol{\alpha}(j)} \in \mathbb{R}_{>0}, b_{\boldsymbol{\beta}} \in \mathbb{R}$, exponents $\boldsymbol{\alpha}(j) \in(2 \mathbb{Z})^{n}, \boldsymbol{\beta} \in \mathbb{Z}^{n}$, such that the following condition holds: there exist unique, positive barycentric coordinates $\lambda_{j}$ relative to the $\boldsymbol{\alpha}(j)$ with $j=1, \ldots, r$ satisfying

$$
\boldsymbol{\beta}=\sum_{j=1}^{r} \lambda_{j} \boldsymbol{\alpha}(j) \text { with } \lambda_{j}>0 \text { and } \sum_{j=1}^{r} \lambda_{j}=1 \text {. }
$$

For every circuit polynomial $p$ we define the corresponding circuit number as

$$
\Theta_{p}=\prod_{j=1}^{r}\left(\frac{b_{\boldsymbol{\alpha}(j)}}{\lambda_{j}}\right)^{\lambda_{j}}
$$

Condition (2.1) implies that $\boldsymbol{A}(p)$ forms a minimal affine dependent set. Those sets are called circuits, see e.g., Oxl11. More specifically, Condition (2.1) yields that $\operatorname{New}(p)$ is a simplex with even vertices $\boldsymbol{\alpha}(1), \ldots, \boldsymbol{\alpha}(r)$ and that the exponent $\boldsymbol{\beta}$ is in the strict interior of $\operatorname{New}(p)$ if $\operatorname{dim}(\operatorname{New}(p)) \geq 1$. Therefore, we call $p_{\boldsymbol{\beta}} \boldsymbol{x}^{\boldsymbol{\beta}}$ the inner term of $p$.

Circuit polynomials are proper building blocks for nonnegativity certificates since the circuit number alone determines whether they are nonnegative.

Theorem 2.2 ([IdW16], Theorem 3.8). Let p be a circuit polynomial as in Definition 2.1. Then $p$ is nonnegative if and only if:

(1) $p$ is a sum of monomial squares, or 
(2) the coefficient $b_{\boldsymbol{\beta}}$ of the inner term of $p$ satisfies $\left|b_{\boldsymbol{\beta}}\right| \leq \Theta_{p}$.

The set of sums of nonnegative circuit polynomials (SONC) is a convex cone. For further details about SONC see dW15, IdW16, DIdW17.

Let us consider $p=\operatorname{poly}(\boldsymbol{A}, \boldsymbol{b})=\sum_{\boldsymbol{\alpha} \in A} b_{\boldsymbol{\alpha}} \boldsymbol{x}^{\boldsymbol{\alpha}}$. To algorithmically determine a lower bound of $p$ via SONC, we take an approach, similar to the one described in SdW18a, $\S 3]$. As initial relaxation, each monomial non-square is equipped with a negative sign. This allows us, to restrict ourselves to the positive orthant; see e.g., [IdW16, Section 3.1] for further details. Next, we compute a covering Cov, which is a sequence of sets $\left(\mathrm{Cov}^{\boldsymbol{\beta}}\right)_{\boldsymbol{\beta} \in \operatorname{NoSq}(p)} \subseteq A$ such that $\mathrm{NoSq}(p) \subseteq \bigcup_{\boldsymbol{\beta} \in \mathrm{NoSq}(p)} \mathrm{Cov}^{\boldsymbol{\beta}}$ and each $\mathrm{Cov}^{\boldsymbol{\beta}}$ is the support of a nonnegative circuit polynomial $p_{\boldsymbol{\beta}}$ with interior point $\boldsymbol{\beta}$. To obtain a covering, we write each non-square as a minimal convex combination of monomial squares, by solving the following LP for each $\boldsymbol{\beta} \in \operatorname{NoSq}(p)$, as explained in [SdW18a, Algorithm 3.3].

$$
\begin{aligned}
\sum_{\boldsymbol{\alpha} \in \operatorname{MoSq}(p)} \lambda_{\boldsymbol{\alpha}}^{\boldsymbol{\beta}} \cdot \boldsymbol{\alpha} & =\boldsymbol{\beta} \\
\sum_{\boldsymbol{\alpha} \in \operatorname{MoSq}(p)} \lambda_{\boldsymbol{\alpha}}^{\boldsymbol{\beta}} & =1 \\
\lambda_{\boldsymbol{\alpha}}^{\boldsymbol{\beta}} & \geq 0 \quad \text { for all } \boldsymbol{\alpha} \in \operatorname{MoSq}(p)
\end{aligned}
$$

If $\left\{\boldsymbol{\alpha}: \lambda_{\boldsymbol{\alpha}}>0\right\}$ is not minimal, then we can reduce it by applying the following lemma. The lemma is folklore, for a constructive proof see e.g. [SdW18a, Lemma 3.1].

Lemma 2.3. For every non-extremal point $\boldsymbol{v} \in A(p) \backslash \operatorname{Vert}(p)$, we can efficiently compute affinely independent $\boldsymbol{v}_{0}, \ldots, \boldsymbol{v}_{m} \in \operatorname{Vert}(p)$ with $m \leq n$ such that and $\boldsymbol{v} \in$ $\operatorname{conv}\left(\left\{\boldsymbol{v}_{0}, \ldots, \boldsymbol{v}_{m}\right\}\right)$.

So for each $\boldsymbol{\beta}$, we obtain a vector $\boldsymbol{\lambda}^{\boldsymbol{\beta}}$ of barycentric coordinates, relative to the simplex $\mathrm{Cov}^{\boldsymbol{\beta}}=\left\{\boldsymbol{\alpha} \in \operatorname{MoSq}(p): \lambda_{\boldsymbol{\alpha}}^{\boldsymbol{\beta}}>0\right\}$. We denote these computations by $\boldsymbol{\lambda},(\mathrm{Cov}):=\operatorname{cover}(p)$.

Then, we solve the following geometric program (GP):

$(\mathrm{SONC})$

$$
\begin{aligned}
p_{\mathrm{SONC}}=\min _{\boldsymbol{X}} & \sum_{\boldsymbol{\beta} \in \operatorname{NoSq}(p)} X_{\boldsymbol{\beta}, \boldsymbol{0}} \\
\text { s.t. } & \sum_{\boldsymbol{\beta} \in \operatorname{NoSq}(p)} X_{\boldsymbol{\beta}, \boldsymbol{\alpha}} \leq b_{\boldsymbol{\alpha}}, \quad \boldsymbol{\alpha} \in \operatorname{MoSq}(p), \boldsymbol{\alpha} \neq \mathbf{0}, \\
& \prod_{\boldsymbol{\alpha} \in \mathrm{Cov}^{\boldsymbol{\beta}}}\left(\frac{X_{\boldsymbol{\beta}, \boldsymbol{\alpha}}}{\lambda_{\boldsymbol{\alpha}}^{\boldsymbol{\beta}}}\right)^{\lambda_{\boldsymbol{\alpha}}}=-b_{\boldsymbol{\beta}}, \quad \boldsymbol{\beta} \in \operatorname{NoSq}(p), \\
& X_{\boldsymbol{\beta}, \boldsymbol{\alpha}} \geq 0, \quad \boldsymbol{\alpha} \in \operatorname{MoSq}(p), \boldsymbol{\beta} \in \operatorname{NoSq}(p) .
\end{aligned}
$$

For an overview about GPs, see BKVH07, BV04. If $p_{\mathrm{SONC}}$ is attained at $\boldsymbol{X}^{\star}$, then one has $p_{\boldsymbol{\beta}}=\sum_{\boldsymbol{\alpha} \in \mathrm{Cov}^{\boldsymbol{\beta}}} X_{\boldsymbol{\beta}, \boldsymbol{\alpha}}^{\star} \cdot \boldsymbol{x}^{\boldsymbol{\alpha}}+b_{\boldsymbol{\beta}} \boldsymbol{x}^{\boldsymbol{\beta}} \geq 0$ by Theorem 2.2, and $p+p_{\mathrm{SONC}}-b_{\mathbf{0}}=$ $\sum_{\boldsymbol{\beta} \in \mathrm{NoSq}(p)} p_{\boldsymbol{\beta}} \geq 0$. Hence, $b_{\mathbf{0}}-p_{\mathrm{SONC}}$ is a lower bound of $p$ on $\mathbb{R}^{n}$. 
These computations correspond to [SdW18a, $\S 3.3 .2$ ], where each cover contains just a single non-square. The approach dispayed here simplifies the computations, while keeping the quality of the results.

2.2. SAGE Polynomials. Let $e:=\exp (1)$. The relative entropy function is defined for $\boldsymbol{\nu}, \boldsymbol{c} \in \mathbb{R}_{+}^{t}$ by $D(\boldsymbol{\nu}, \boldsymbol{c}):=\sum_{j=1}^{t} \nu_{j} \log \frac{\nu_{j}}{c_{j}}$. A signomial $p$ is a weighted sum of exponentials composed with linear functionals of a variable $\boldsymbol{x} \in \mathbb{R}^{n}$ : given $t \in \mathbb{N}, c_{1}, \ldots, c_{t} \in \mathbb{Q}$ and $\boldsymbol{\alpha}(1), \ldots, \boldsymbol{\alpha}(t) \in \mathbb{N}^{n}$, we write $p(\boldsymbol{x})=\sum_{j=1}^{t} c_{j} \exp (\boldsymbol{\alpha}(j) \cdot \boldsymbol{x})$. Note that for general signomials, one considers $c_{1}, \ldots, c_{t} \in \mathbb{R}$ and $\boldsymbol{\alpha}(1), \ldots, \boldsymbol{\alpha}(t) \in \mathbb{R}^{n}$. However, for certification purposes, we restrict the coefficients to the set of rationals and the exponents to tuples of nonnegative integers. A globally nonnegative signomial with at most one negative coefficient is called an $A M / G M$ exponential or arithmetic-geometric-mean-exponential (AGE). Certifying the nonnegativity of an AGE is done by verifying an arithmetic-geometric-mean inequality. This is recalled in the following result, stated in [CS16, Lemma 2.2].

Lemma 2.4. Let $p(\boldsymbol{x})=\sum_{j=1}^{t} c_{j} \exp (\boldsymbol{\alpha}(j) \cdot \boldsymbol{x})+\beta \exp (\boldsymbol{\alpha}(0) \cdot \boldsymbol{x})$, with $c_{1}, \ldots, c_{t} \in \mathbb{Q}_{>0}$, $\beta \in \mathbb{Q}$ and $\boldsymbol{\alpha}(0), \boldsymbol{\alpha}(1), \ldots, \boldsymbol{\alpha}(t) \in \mathbb{N}^{n}$. Then $p(\boldsymbol{x}) \geq 0$ for all $\boldsymbol{x} \in \mathbb{R}^{n}$ if and only if there exists $\boldsymbol{\nu} \in \mathbb{R}_{+}^{t}$ such that $D(\boldsymbol{\nu}, e \boldsymbol{c}) \leq \beta$ and $\sum_{j=1}^{t} \boldsymbol{\alpha}(j) \nu_{j}=(\mathbf{1} \cdot \boldsymbol{\nu}) \boldsymbol{\alpha}(0)$.

Given $\boldsymbol{\alpha}(0), \boldsymbol{\alpha}(1), \ldots, \boldsymbol{\alpha}(t) \in \mathbb{N}^{n}$, the set of AGE signomials is a convex cone, denoted by $\mathcal{C}_{\mathrm{AGE}}$, and defined as follows:

$\mathcal{C}_{\mathrm{AGE}}:=\left\{(\boldsymbol{c}, \beta) \in \mathbb{R}_{+}^{t} \times \mathbb{R}:\right.$ There exists $\boldsymbol{\nu} \in \mathbb{R}_{+}^{t}$ with $\left.D(\boldsymbol{\nu}, e \boldsymbol{c}) \leq \beta, \sum_{j=1}^{t} \boldsymbol{\alpha}(j) \nu_{j}=(\mathbf{1} \cdot \boldsymbol{\nu}) \boldsymbol{\alpha}(0)\right\}$.

The set of sums of $A G E$ ( $S A G E$ ) polynomials is also a convex cone, denoted by $\mathcal{C}_{\mathrm{SAGE}}$. By [CS16, Proposition 2.4], one has the following characterisation.

Theorem 2.5. A signomial $f=\sum_{i=1}^{t} b_{j} \exp (\boldsymbol{\alpha}(j) \cdot \boldsymbol{x})$ lies in $\mathcal{C}_{S A G E}$ if and only if there is $\boldsymbol{c}^{(1)}, \ldots, \boldsymbol{c}^{(t)}, \boldsymbol{\nu}^{(1)}, \ldots, \boldsymbol{\nu}^{(t)} \in \mathbb{R}^{t}$ satisfying the following conditions:

(SAGE-feas)

$$
\begin{gathered}
\sum_{j=1}^{t} \boldsymbol{c}^{(j)}=\boldsymbol{b}, \quad \sum_{i=1}^{t} \boldsymbol{\alpha}(i) \boldsymbol{\nu}_{i}^{(j)}=\mathbf{0}, \quad-\mathbf{1} \cdot \boldsymbol{\nu}_{\backslash j}^{(j)}=\nu_{j}^{(j)}, \\
\boldsymbol{c}_{\backslash j}^{(j)}, \boldsymbol{\nu}_{\backslash j}^{(j)} \geq \mathbf{0}, \quad D\left(\boldsymbol{\nu}_{\backslash j}^{(j)}, e \boldsymbol{c}_{\backslash j}^{(j)}\right) \leq c_{j}^{(j)}, \quad j=1, \ldots, t .
\end{gathered}
$$

One way to obtain lower bounds of a signomial $f$ is to solve the following REP:

$$
f_{\mathrm{SAGE}}=\sup \left\{C \in \mathbb{R}: f-C \in \mathcal{C}_{\mathrm{SAGE}}\right\} .
$$

The constraints of (SAGE) correspond to (SAGE-feas), after replacing $\boldsymbol{b}$ by the vector of coefficients of $f-\lambda$.

\section{Exact Optimization via SONC/SAGE}

In this section, we present two algorithms for converting a numerical solution for SONC and SAGE into a lower bound in exact arithmetic. For a polynomial $p=\sum_{\boldsymbol{\alpha} \in A} b_{\boldsymbol{\alpha}} \boldsymbol{x}^{\boldsymbol{\alpha}}$, we assume $\mathbf{0} \in A$, so there exists a constant term $p(\mathbf{0}) \neq 0$. Furthermore, we require 
that every non-square monomial lies in the interior of New $(p)$ or on a face of New $(p)$ including the origin. We denote numerical solutions $\widetilde{\boldsymbol{X}}, \widetilde{\boldsymbol{c}}, \widetilde{\boldsymbol{\nu}}$ with a tilde, intermediate rational solutions $\widehat{\boldsymbol{X}}, \widehat{\boldsymbol{c}}$ with a hat, and our final rational solution with regular letters.

3.1. Symbolic Post-Processing for SONC. We focus on certifying exactly lower bounds of a given polynomial via SONC decompositions. We rely on the numerical procedure from Section 2.1, which starts to compute a covering of this polynomial. Under these assumptions, we design an algorithm, called opts onc, to convert numerical lower bounds, corresponding to SONC decompositions obtained via GP, into exact lower bounds.

Algorithm 3.1. optsonc

Require: $p=\sum_{\boldsymbol{\alpha} \in A} b_{\boldsymbol{\alpha}} \boldsymbol{x}^{\boldsymbol{\alpha}} \in \mathbb{Q}[\boldsymbol{x}]$, rounding precision $\widehat{\delta} \in \mathbb{Q}_{>0}$, precision parameter $\widetilde{\delta} \in \mathbb{Q}_{>0}$ for the GP solver.

Ensure: Matrix $\boldsymbol{X}$ of rational numbers, coefficients of the decomposition, certified lower bound $C \in \mathbb{Q}$ of $p$ on $\mathbb{R}^{n}$.

1: $(\boldsymbol{\lambda}, \operatorname{Cov}) \leftarrow \operatorname{cover}(p)$

2: $\widetilde{\boldsymbol{X}} \leftarrow \operatorname{GP}(p, \widetilde{\delta}, \boldsymbol{\lambda}, \mathrm{Cov})$

3: $\widehat{\boldsymbol{X}} \leftarrow \operatorname{round}(\widetilde{\boldsymbol{X}}, \widehat{\delta})$

$\triangleright$ Solve (SONC) with accuracy $\widetilde{\delta}$

$\triangleright$ rounding step

4: for $\boldsymbol{\alpha} \in \operatorname{MoSq}(p)$ and $\boldsymbol{\beta} \in \operatorname{NoSq}(p)$ do

5: $\quad X_{\boldsymbol{\beta}, \boldsymbol{\alpha}} \leftarrow b_{\boldsymbol{\alpha}} \cdot \widehat{X}_{\boldsymbol{\beta}, \boldsymbol{\alpha}} / \sum_{\boldsymbol{\beta}^{\prime} \in \mathrm{NoSq}(p)} \widehat{X}_{\boldsymbol{\beta}^{\prime}, \boldsymbol{\alpha}} \quad \triangleright$ projection step

6: end for

7: for $\boldsymbol{\beta} \in \operatorname{NoSq}(p)$ do

8: $\quad$ coeff $=\lambda_{\mathbf{0}}^{\boldsymbol{\beta}} \cdot\left(-b_{\boldsymbol{\beta}} \cdot \prod_{\boldsymbol{\alpha} \in \operatorname{Cov}^{\beta}}\left(\frac{\lambda_{\boldsymbol{\alpha}}^{\boldsymbol{\beta}}}{X_{\boldsymbol{\beta}, \alpha}}\right)^{\lambda_{\boldsymbol{\alpha}}^{\boldsymbol{\beta}}}\right)^{\frac{1}{\lambda_{\mathbf{0}}^{\boldsymbol{\beta}}}}$

9: $\quad X_{\beta, 0} \leftarrow$ round-up $($ coeff,,$\widehat{\delta})$

$\triangleright$ adjust constant term

10: end for

11: $C \leftarrow b_{\mathbf{0}}-\sum_{\boldsymbol{\beta} \in \operatorname{NoSq}(p)} X_{\boldsymbol{\beta}, \mathbf{0}}$

12: return $X, C$

In line 2, the function GP calls a GP solver to compute a $\widetilde{\delta}$-approximation $\widetilde{\boldsymbol{X}}$ of $($ SONC) This approximation is then rounded in line 3 to a rational point $\widehat{\boldsymbol{X}}$ with a prescribed maximal relative error of $\widehat{\delta}$. The projection step from line 5 scales the entries of $\widehat{\boldsymbol{X}}$, yielding $\sum_{\boldsymbol{\beta}} X_{\boldsymbol{\beta}, \boldsymbol{\alpha}}=b_{\boldsymbol{\alpha}}$, for all $\boldsymbol{\beta} \in \operatorname{MoSq}(p)$, to satisfy the first set of equality constraints of (SONC) In line 9, we round the coefficient up, with relative error $\widehat{\delta}$, so that we have

$$
X_{\boldsymbol{\beta}, \mathbf{0}} \geq \lambda_{\mathbf{0}}^{\boldsymbol{\beta}} \cdot\left(-b_{\boldsymbol{\beta}} \cdot \prod_{\boldsymbol{\alpha} \in \mathrm{Cov}^{\boldsymbol{\beta}}}\left(\frac{\lambda_{\boldsymbol{\alpha}}^{\boldsymbol{\beta}}}{X_{\boldsymbol{\beta}, \boldsymbol{\alpha}}}\right)^{\lambda_{\boldsymbol{\alpha}}^{\beta}}\right)^{\frac{1}{\lambda_{0}^{\beta}}}
$$

As in Section 2.1, each $p_{\boldsymbol{\beta}}:=\sum_{\boldsymbol{\alpha} \in \mathrm{Cov}^{\boldsymbol{\beta}}} X_{\boldsymbol{\beta}, \boldsymbol{\alpha}} \cdot \boldsymbol{x}^{\boldsymbol{\alpha}}+b_{\beta} \boldsymbol{x}^{\boldsymbol{\beta}}$ is a nonnegative circuit polynomial. Hence, $C$ is a lower bound for $p$. Our assumption that every circuit polynomial contains a constant term, is necessary to ensure that $\lambda_{\mathbf{0}}^{\boldsymbol{\beta}} \neq 0$ for all $\boldsymbol{\beta} \in \operatorname{NoSq}(p)$ in our computations above. 
3.2. Symbolic Post-Processing for SAGE. Similarly to Algorithm 3.1, our algorithm optsage takes a given polynomial as input, obtains a numerical lower bound related to a SAGE decomposition computed via REP, and applies a post-processing to find a certified lower bound .

\section{Algorithm 3.2. optsage}

Require: $g=\sum_{i=1}^{t} b_{i} \boldsymbol{x}^{\alpha(i)} \in \mathbb{Q}[\boldsymbol{x}]$, rounding precision $\widehat{\delta} \in \mathbb{Q}_{>0}$, precision parameter $\widetilde{\delta} \in \mathbb{Q}_{>0}$ for the REP solver.

Ensure: Matrices $\boldsymbol{c}, \boldsymbol{\nu}$ of rational numbers, coefficients of the decomposition, certified lower bound $C \in \mathbb{Q}$ of $g$ on $\mathbb{R}^{n}$.

1: $f \leftarrow g(\exp \boldsymbol{x}-\exp (-\boldsymbol{x}))$

2: Build the $(n+1) \times t$ matrix $\boldsymbol{Q}$ with columns $(\boldsymbol{\alpha}(1), 1), \ldots,(\boldsymbol{\alpha}(t), 1)$

3: $\widetilde{\boldsymbol{c}}, \widetilde{\boldsymbol{\nu}} \leftarrow \operatorname{REP}(f, \widetilde{\delta})$

4: $\widehat{\boldsymbol{c}} \leftarrow \operatorname{round}(\widetilde{\boldsymbol{c}}, \widehat{\delta}), \quad \widehat{\boldsymbol{\nu}} \leftarrow \operatorname{round}(\widetilde{\boldsymbol{\nu}}, \widehat{\delta})$ $\triangleright$ Solve (SAGE) with accuracy $\widetilde{\delta}$

5: for $j \in\{1, \ldots, t\}$ do

6: $\quad \mathrm{LP} \leftarrow\left\{\boldsymbol{Q} \cdot \boldsymbol{\nu}^{(j)}=\mathbf{0}, \boldsymbol{\nu}_{\backslash j}^{(j)} \geq \mathbf{0},\left\|\boldsymbol{\nu}^{(j)}-\widetilde{\boldsymbol{\nu}}^{(j)}\right\|_{\infty} \leq \widehat{\delta}, \nu_{1}^{(j)} \geq \widehat{\delta}\right\}$

7: $\quad \boldsymbol{\nu}^{(j)} \leftarrow$ some element from LP

8: $\quad \boldsymbol{c}_{\backslash j}^{(j)} \leftarrow \widehat{\boldsymbol{c}}_{\backslash j}^{(j)}, c_{j}^{(j)} \leftarrow b_{j}-\mathbf{1} \cdot \boldsymbol{c}_{\backslash j}^{(j)}$

$\triangleright$ projection step

9: end for

10: for $j \in\{1, \ldots, t\}$ do

11: $\quad$ power $\leftarrow 1-\log \nu_{1}^{(j)}-\frac{1}{\nu_{1}^{(j)}}\left(c_{j}^{(j)}-\sum_{i>1, i \neq j} \nu_{i}^{(j)} \log \frac{\nu_{i}^{(j)}}{e c_{i}^{(j)}}\right)$

12: $\quad c_{1}^{(j)} \leftarrow$ round-up $(\exp ($ power $), \widehat{\delta})$ $\triangleright$ rounding step

\section{3: end for}

14: $C \leftarrow b_{1}-\sum_{j=1}^{t} c_{1}^{(j)}$

15: return $c, \nu, C$

Given a polynomial $g(\boldsymbol{y})=\sum_{j=1}^{t} b_{j} \boldsymbol{y}^{\boldsymbol{\alpha}(j)}$, one could apply the change of variables $y_{i}:=$ $\exp x_{i}$ when $\boldsymbol{y} \in \mathbb{R}_{>0}^{n}$. Since this transformation is only valid on the nonnegative orthant, one workaround used in optsage is to define the signomial $f(\boldsymbol{x})=g(\exp \boldsymbol{x}-\exp (-\boldsymbol{x}))$ from line 1, in a such a way that a lower bound of $f$ yields a lower bound of $g$. The REP function in line 3 calls an REP solver to compute a $\widetilde{\delta}$-approximation $(\widetilde{\boldsymbol{\nu}}, \widetilde{\boldsymbol{c}})$ of (SAGE). This approximation is then rounded to a rational point $(\widehat{\boldsymbol{\nu}}, \widehat{\boldsymbol{c}})$ with a prescribed maximal relative error of $\widehat{\delta}$. The projection steps in line 7 and line 8 ensure that $(\boldsymbol{\nu}, \boldsymbol{c})$ satisfies exactly the linear equality constraints of (SAGE) i.e., $\boldsymbol{Q} \boldsymbol{\nu}^{(j)}=\mathbf{0}$ and $\sum_{j=1}^{t} \boldsymbol{c}^{(j)}=b$. The first projection step boils down to exactly solve an LP with the constraint that $\nu_{1}^{(j)}>0$, for all $j=1, \ldots, t$, to ensure that further computation in line 12 are well-defined. Note that this projection could be done while relying on the pseudo-inverse of $\boldsymbol{Q}$, but one obtains better practical results via this procedure. To ensure that the relative entropy inequality constraints of (SAGE) are satisfied, the last step of optsage aims at finding $c_{j}^{(1)}$ such that $c_{j}^{(j)} \geq D\left(\boldsymbol{\nu}_{\backslash j}^{(j)}, e \boldsymbol{c}_{\backslash j}^{(j)}\right)=$ 
$\sum_{i>1, i \neq j} \nu_{i}^{(j)} \log \frac{\nu_{i}^{(j)}}{e c_{i}^{(j)}}+\nu_{1}^{(j)} \log \frac{\nu_{1}^{(j)}}{e c_{1}^{(j)}}$. Thus, one relies on the round-up procedure in line 12 to compute $c_{1}^{(j)} \geq \exp \left(1-\log \nu_{1}^{(j)}-\frac{1}{\nu_{1}^{(j)}}\left(c_{j}^{(j)}-\sum_{i>1, i \neq j} \nu_{i}^{(j)} \log \frac{\nu_{i}^{(j)}}{e c_{i}^{(j)}}\right)\right)$. Eventually, one has $\sum_{j=1}^{t} c_{i}^{(j)}=b_{i}$, for all $i>1$ and $\sum_{j=1}^{t} c_{1}^{(j)}=b_{1}-C$, which certifies that $f-C \geq 0$ on $\mathbb{R}^{n}$. We refer to appendix A.2 for an example of exact SAGE decomposition obtained with optsage.

\section{Deciding Nonnegativity via SAGE}

We denote by int $\left(\mathcal{C}_{\mathrm{SAGE}}\right)$ the interior of the cone $\mathcal{C}_{\mathrm{SAGE}}$ of SAGE signomials. A signomial $f=\sum_{j=1}^{t} b_{j} \exp (\boldsymbol{\alpha}(j) \cdot \boldsymbol{x})$ lies in int $\left(\mathcal{C}_{\mathrm{SAGE}}\right)$ if and only there is $\boldsymbol{c}^{(1)}, \ldots, \boldsymbol{c}^{(t)}$, $\boldsymbol{\nu}^{(1)}, \ldots, \boldsymbol{\nu}^{(t)} \in \mathbb{R}^{t}$ such that

(INTSAGE-feas)

$$
\begin{gathered}
\sum_{j=1}^{t} \boldsymbol{c}^{(j)}=\boldsymbol{b}, \quad \sum_{i=1}^{t} \boldsymbol{\alpha}(i) \boldsymbol{\nu}_{i}^{(j)}=\mathbf{0}, \quad-\mathbf{1} \cdot \boldsymbol{\nu}_{\backslash j}^{(j)}=\nu_{j}^{(j)}, \\
\boldsymbol{c}_{\backslash j}^{(j)}, \boldsymbol{\nu}_{\backslash j}^{(j)}>\mathbf{0}, \quad D\left(\boldsymbol{\nu}_{\backslash j}^{(j)}, e \boldsymbol{c}_{\backslash j}^{(j)}\right)<c_{j}^{(j)}, \quad j=1, \ldots, t .
\end{gathered}
$$

Without the assumptions from Section 3, we state and analyze a decision algorithm to certify nonnegativity of signomials belonging to the interior int $\left(\mathcal{C}_{\mathrm{SAGE}}\right)$ of the SAGE cone. The resulting hybrid numeric-symbolic algorithm, called intsage, computes exact rational SAGE decompositions of such signomials.

For complexity analysis purpose, we recall the following bound for the roots of univariate polynomials with integer coefficients:

Lemma 4.1. Mig92, Theorem 4.2 (ii)] Let $f \in \mathbb{Z}[x]$ of degree $d$, with coefficient bit size bounded from above by $\tau$. If $f(\widetilde{x})=0$ and $\widetilde{x} \neq 0$, then $\frac{1}{2^{\tau}+1} \leq|\widetilde{x}| \leq 2^{\tau}+1$.

Lemma 4.2. Let $f=\sum_{j=1}^{t} b_{j} \exp (\boldsymbol{\alpha}(j) \cdot \boldsymbol{x}) \in \operatorname{int}\left(\mathcal{C}_{S A G E}\right)$ of degree $d$ with $\tau=\tau(f)$. Then, there exists $N \in \mathbb{N}$ such that for $\varepsilon:=2^{-N}, f-\varepsilon \sum_{j=1}^{t} \exp (\boldsymbol{\alpha}(j) \cdot \boldsymbol{x}) \in \mathcal{C}_{S A G E}$, with $N \leq \tau(\varepsilon) \in \mathcal{O}\left(\tau \cdot(4 d+6)^{3 n+3}\right)$.

Proof. Since $f \in \operatorname{int}\left(\mathcal{C}_{\mathrm{SAGE}}\right)$, there are $\boldsymbol{c}^{(1)}, \ldots, \boldsymbol{c}^{(t)}, \boldsymbol{\nu}^{(1)}, \ldots, \boldsymbol{\nu}^{(t)} \in \mathbb{R}^{t}$ such that $\sum_{j=1}^{t} \boldsymbol{c}^{(j)}=\boldsymbol{b}, \sum_{i=1}^{t} \boldsymbol{\alpha}(i) \boldsymbol{\nu}_{i}^{(j)}=\mathbf{0},-\mathbf{1} \cdot \boldsymbol{\nu}_{\backslash j}^{(j)}=\nu_{j}^{(j)}, \boldsymbol{c}_{\backslash j}^{(j)}, \boldsymbol{\nu}_{\backslash j}^{(j)}>\mathbf{0}$ and $D\left(\boldsymbol{\nu}_{\backslash j}^{(j)}, e \boldsymbol{c}_{\backslash j}^{(j)}\right)<c_{j}^{(j)}$, for all $j=1, \ldots, t$. Therefore, there exists $N \in \mathbb{N}$ such that for $\varepsilon:=2^{-N}$, one has $D\left(\boldsymbol{\nu}_{\backslash j}^{(j)}, e \boldsymbol{c}_{\backslash j}^{(j)}\right)+\varepsilon<c_{j}^{(j)}$, for all $j=1, \ldots, t$. For all $i, j=1, \ldots, t$, let us define $\stackrel{b}{b}^{\circ}$ by $\stackrel{\circ}{b}_{i}:=b_{i}-\varepsilon$, as well as $\stackrel{\circ}{c}$ by $\stackrel{\circ}{c}_{i}^{(j)}:=c_{i}^{(j)}$ for $i \neq j$ and $\stackrel{\circ}{c}_{j}^{(j)}:=c_{j}^{(j)}-\varepsilon$. Note that

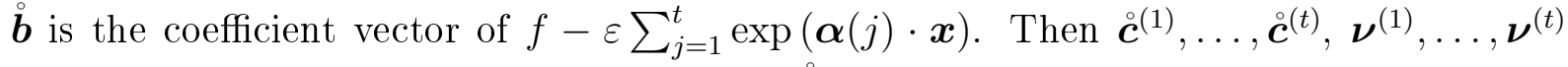
satisfy (INTSAGE-feas) after replacing $\boldsymbol{b}$ by $\boldsymbol{b}$, yielding the first claim.

For the second claim, we start to perform the change of variable $y_{i}:=\exp x_{i}$, for all $i=1, \ldots, n$, define $g(\boldsymbol{y}, z):=\sum_{j=1}^{t} b_{j} \boldsymbol{y}^{\boldsymbol{\alpha}(j)}-z \sum_{j=1}^{t} \boldsymbol{y}^{\boldsymbol{\alpha}(j)}$, for all $\boldsymbol{y} \in \mathbb{R}_{>0}^{n}$. It is enough to select $\varepsilon=2^{-N}$ such that $\varepsilon \leq \inf _{\boldsymbol{y} \in \mathbb{R}_{>0}^{n}} \frac{\sum_{j=1}^{t} b_{j} \boldsymbol{y}^{\alpha(j)}}{\sum_{j=1}^{t} \boldsymbol{y}^{\alpha(j)}}$. Let us consider the algebraic set $V$ 
defined by:

$$
V:=\left\{(\boldsymbol{y}, z) \in \mathbb{R}^{n+1}: g(\boldsymbol{y}, z)=\frac{\partial g}{\partial y_{1}}=\cdots=\frac{\partial g}{\partial y_{n}}=0\right\} .
$$

Using [MSED18, Proposition A.1], there exists a polynomial in $\mathbb{Z}[z]$ of degree less than $(d+1)^{n+1}$ with coefficients of bit size less than $\tau \cdot(4 d+6)^{3 n+3}$ such that its set of real roots contains $V$. By Lemma 4.1, it is enough to take $N \leq \tau \cdot(4 d+6)^{3 n+3}$, yielding the desired result.

4.1. Algorithm intsage. We present our algorithm intsage computing exact rational SAGE decompositions for signomials in $\operatorname{int}\left(\mathcal{C}_{\mathrm{SAGE}}\right)$.

\section{Algorithm 4.3. intsage}

Require: $f=\sum_{j=1}^{t} b_{j} \exp (\boldsymbol{\alpha}(j) \cdot \boldsymbol{x}) \in \operatorname{int}\left(\mathcal{C}_{\mathrm{SAGE}}\right)$, rounding precision $\widehat{\delta} \in \mathbb{Q}_{>0}$, precision parameter $\widetilde{\delta} \in \mathbb{Q}_{>0}$ for the REP solver.

Ensure: Matrices $\boldsymbol{c}, \boldsymbol{\nu}$ of rational numbers.

1: Build the $(n+1) \times t$ matrix $\boldsymbol{Q}$ with columns $(\boldsymbol{\alpha}(1), 1), \ldots,(\boldsymbol{\alpha}(t), 1)$

2: $\boldsymbol{Q}^{+} \leftarrow \operatorname{pseudoinv}(\boldsymbol{Q})$

$3:$ ok $\leftarrow$ false

4: while not ok do

5: $\quad(\widetilde{\boldsymbol{c}}, \widetilde{\boldsymbol{\nu}}) \leftarrow \operatorname{REP}(f, \widetilde{\delta})$

6: $\quad \widehat{\boldsymbol{c}} \leftarrow \operatorname{round}(\widetilde{\boldsymbol{c}}, \widehat{\delta}), \quad \widehat{\boldsymbol{\nu}} \leftarrow \operatorname{round}(\widetilde{\boldsymbol{\nu}}, \widehat{\delta})$

7: $\quad$ for $j \in\{1, \ldots, t\}$ do

8: $\quad \boldsymbol{\nu}^{(j)} \leftarrow\left(\boldsymbol{I}-\boldsymbol{Q}^{+} \boldsymbol{Q}\right) \widehat{\boldsymbol{\nu}}^{(j)}$

9: $\quad \boldsymbol{c}_{\backslash j}^{(j)} \leftarrow \widehat{\boldsymbol{c}}_{\backslash j}^{(j)}, \quad c_{j}^{(j)} \leftarrow b_{j}-\mathbf{1} \cdot \boldsymbol{c}_{\backslash j}^{(j)}$

10: $\quad$ end for

11: $\quad$ if for all $j \in\{1, \ldots, t\}, \boldsymbol{\nu}_{\backslash j}^{(j)}, \boldsymbol{c}_{\backslash j}^{(j)} \geq \mathbf{0}, c_{j}^{(j)} \geq D\left(\boldsymbol{\nu}_{\backslash j}^{(j)}, e \boldsymbol{c}_{\backslash j}^{(j)}\right)$, then ok $\leftarrow$ true $\quad \triangleright$ verification step

12: $\quad$ else $\widetilde{\delta} \leftarrow \widetilde{\delta} / 2, \widehat{\delta} \leftarrow \widehat{\delta} / 2$

13: $\quad$ end if

14: end while

15: return $c, \nu$

The routine pseudoinv in line 2 computes the pseudo-inverse of $\boldsymbol{Q}$, i.e., a matrix $\boldsymbol{Q}^{+}$such that $\boldsymbol{Q} \boldsymbol{Q}^{+} \boldsymbol{Q}=\boldsymbol{Q}$. Next, we enter in the loop starting from line 4 . The REP function calls an REP solver to compute a $\widetilde{\delta}$-approximation $(\widetilde{\boldsymbol{\nu}}, \widetilde{\boldsymbol{c}})$ of (INTSAGE-feas). The projection steps ensure that $(\boldsymbol{\nu}, \boldsymbol{c})$ satisfies exactly the linear equality constraints of (SAGE-feas), i.e., $\boldsymbol{Q} \boldsymbol{\nu}^{(j)}=\boldsymbol{Q}\left(\boldsymbol{I}-\boldsymbol{Q}^{+} \boldsymbol{Q}\right) \boldsymbol{\nu}^{(j)}=\boldsymbol{Q}-\boldsymbol{Q} \boldsymbol{Q}^{+} \boldsymbol{Q}=\mathbf{0}$ and $\sum_{j=1}^{t} \boldsymbol{c}^{(j)}=b$. If the inequality constraints are not verified in line 11, the rounding-projection procedure is performed again with more accuracy.

4.2. Arithmetic Complexity. Before analyzing the arithmetic complexity of intsage, we first establish lower bounds for the nonnegative components of the solutions related to SAGE decompositions of polynomials in int $\left(\mathcal{C}_{\mathrm{SAGE}}\right)$. 
Lemma 4.4. Let $f=\sum_{j=1}^{t} b_{j} \exp (\boldsymbol{\alpha}(j) \cdot \boldsymbol{x}) \in \operatorname{int}\left(\mathcal{C}_{S A G E}\right)$ of degree $d$ with $\tau=\tau(f)$. Let $\varepsilon$ be as in Lemma 4.2.

(1) There exists a solution of $(\boldsymbol{\nu}, \boldsymbol{c})$ of (INTSAGE-feas) and $\delta \in \mathbb{Q}_{>0}$ such that $\delta \leq 1$, $(\boldsymbol{\nu}, \boldsymbol{c})$ satisfies, $D\left(\boldsymbol{\nu}_{\backslash j}^{(j)}, e\left(\boldsymbol{c}_{\backslash j}^{(j)}+\delta \mathbf{1}\right)\right)+\frac{\varepsilon}{2} \leq c_{j}^{(j)}, \sum_{j=1}^{t} \boldsymbol{c}^{(j)}=\boldsymbol{b}$, for all $i, j=$ $1, \ldots, t$.

(2) There exists a solution $(\boldsymbol{\nu}, \boldsymbol{c})$ of $($ INTSAGE-feas $)$ and $\delta \in \mathbb{Q}_{>0}$ such that $(\boldsymbol{\nu}, \boldsymbol{c})$ satisfies $D\left(\boldsymbol{\nu}_{\backslash j}^{(j)}+\delta \mathbf{1}, e \boldsymbol{c}_{\backslash j}^{(j)}\right)+\frac{\varepsilon}{2} \leq c_{j}^{(j)}$, for all $j=1, \ldots, t$.

(3) There exists a solution $(\boldsymbol{\nu}, \boldsymbol{c})$ of $($ INTSAGE-feas $)$ and $\delta \in \mathbb{Q}_{>0}$ such that $(\boldsymbol{\nu}, \boldsymbol{c})$ satisfies $D\left((1+\delta) \boldsymbol{\nu}_{\backslash j}^{(j)}, e \boldsymbol{c}_{\backslash j}^{(j)}\right)+\frac{\varepsilon}{2} \leq c_{j}^{(j)}$, for all $j=1, \ldots, t$.

In each case, $\tau(\delta) \in \mathcal{O}\left(\tau \cdot(4 d+6)^{3 n+3}\right)$.

A proof for this lemma is provided in appendix A.1.

Theorem 4.5. Let $f=\sum_{j=1}^{t} b_{j} \exp (\boldsymbol{\alpha}(j) \cdot \boldsymbol{x}) \in \operatorname{int}\left(\mathcal{C}_{S A G E}\right)$ of degree $d$ and $\tau=\tau(f)$. There exist $\widehat{\delta}$ and $\widetilde{\delta}$ of bit size less than $\mathcal{O}\left(\tau \cdot(4 d+6)^{3 n+3}\right)$, such that intsos $(f, \widehat{\delta}, \widetilde{\delta})$ terminates and outputs a rational SAGE decomposition of $f$ within $\mathcal{O}\left(\tau \cdot(4 d+6)^{3 n+3} t^{7} \log t\right)$ arithmetic operations.

Proof. We first show that the loop of Algorithm intsage terminates with $\widehat{\delta}$ and $\widetilde{\delta}$ of bit size bounded by $\mathcal{O}\left(\tau \cdot(4 d+6)^{3 n+3}\right)$. Let $\varepsilon$ be as in Lemma 4.2 . When running the procedure REP, one solves (SAGE-feas) at precision $\widetilde{\delta}$, thus one finds an approximate solution $(\widetilde{\boldsymbol{\nu}}, \widetilde{\boldsymbol{c}})$ such that $\left\|\sum_{j=1}^{t} \widetilde{\boldsymbol{c}}^{(j)}-\boldsymbol{b}\right\|_{\infty} \leq \widetilde{\delta}, D\left(\widetilde{\boldsymbol{\nu}}_{\backslash j}^{(j)}, e \widetilde{\boldsymbol{c}}_{\backslash j}^{(j)}\right)+\varepsilon \leq \widetilde{c}_{j}^{(j)}+\widetilde{\delta}$, and $\left\|\boldsymbol{Q} \widetilde{\boldsymbol{\nu}}^{(j)}\right\|_{\infty} \leq \widetilde{\delta}$, for all $j=1, \ldots, t$. After the rounding and projection steps, one obtains $\boldsymbol{\nu}^{(j)}=\left(\boldsymbol{I}-\boldsymbol{Q}^{+} \boldsymbol{Q}\right) \widehat{\boldsymbol{\nu}}^{(j)}$ and $\left\|\widehat{\boldsymbol{\nu}}^{(j)}-\widetilde{\boldsymbol{\nu}}^{(j)}\right\|_{\infty} \leq \widehat{\delta}$. Since $\boldsymbol{\alpha}_{j}(i) \leq d$, for all $i, j=1, \ldots, t$, the bit size of the entries of the matrix $\boldsymbol{Q}$ is upper bounded by $\tau(d)$. Thus, the pseudo-inverse $\boldsymbol{Q}^{+}$has rational entries of bit size bounded by $\mathcal{O}(t \log t+t \log d)=\mathcal{O}(t \log t)$, since the bit size is the same as for the determinant length, see [BPR98, Corollary 8.13]. This implies that the bit size of the difference between the entries of $\widetilde{\boldsymbol{\nu}}$ and $\boldsymbol{\nu}$ is upper bounded by $\mathcal{O}(t \log t+\tau(\widetilde{\delta})+\tau(\widehat{\delta}))$. Similarly, the bit size of the difference between the entries of $\widetilde{\boldsymbol{c}}$ and $\boldsymbol{c}$ is upper bounded by $\mathcal{O}(\tau+\tau(\widehat{\delta}))$. By Lemma 4.4 . one can perform any absolute or relative perturbation of $\widetilde{\boldsymbol{\nu}}$ and $\widetilde{\boldsymbol{c}}$, and still ensure that the resulting $(\boldsymbol{\nu}, \boldsymbol{c})$ satisfies $D\left(\boldsymbol{\nu}_{\backslash j}^{(j)}, e \boldsymbol{c}_{\backslash j}^{(j)}\right) \leq c_{j}^{(j)}$, if the perturbation is small enough with bit size at most $\mathcal{O}\left(\tau \cdot(4 d+6)^{3 n+3}\right)$. This implies that one must choose $\widetilde{\delta}$ and $\widehat{\delta}$ small enough, with the same upper bound on their bit sizes. The same reasoning applies to ensure that $\boldsymbol{\nu}_{\backslash j}^{(j)}, \boldsymbol{c}_{\backslash j}^{(j)} \geq \mathbf{0}$.

Now, we give an upper bound on the number of arithmetic operations. For convex optimization problems having barrier complexity equal to $N$, the standard interior-point methods compute a $\widetilde{\delta}$-accurate solution in $\mathcal{O}(\tau(\widetilde{\delta}) \sqrt{N} \log (N))$ iterations; see e.g. Ren01, Section 2.4]. For $(x, y, z) \in \mathbb{R}^{3}$, the standard barrier complexity of a single relative entropy constraint " $x \log (x / y) \leq z, x, y \geq 0$ " is equal to 4 . In addition, the barrier complexity of a set of constraints is upper bounded by the sum of the complexities of the individual 
constraints. Therefore, the relative entropy formulation given in (SAGE-feas) has a barrier complexity of $N \leq 4 t^{2}$. At each iteration of the interior-point method, one needs to solve an LP involving $2 t^{2}$ variables, which can be done within $\mathcal{O}\left(t^{6}\right)$ arithmetic operations. This yields the upper bound of $\mathcal{O}\left(\tau \cdot(4 d+6)^{3 n+3} t^{7} \log t\right)$ on the total number of arithmetic operations required while calling intsage. All other arithmetic operations performed by the algorithm have a negligible cost with respect to the REP procedure.

\section{EXPERIMENTAL COMPARISONS}

We discuss the actual bit sizes and physical running time of optsonc and optsage procedures, given by Algorithm 3.1 and Algorithm 3.2. We describe the setup of our experiment and explain how our random instances were created. Afterwards, we discuss a few selected examples, which exhibit well the differences of the methods, and present how the program behaved on a large set of examples.

5.1. Experimental Setup. We give an overview about the experimental setup.

Software The entire experiment was steered by our PYTHON 3.7 based software POEM 0.2.0.0(a) (Effective Methods in Polynomial Optimization), SdW18b, which we develop since July 2017. POEM is open source, under GNU public license, and available at:

https : //www3.math.tu-berlin.de/combi/RAAGCon0pt/poem.html

For our experiment, POEM calls a range of further software and solvers for computing the certificates. For the numerical solutions of SONC and SAGE, we use CVXPY 1.0.12 [DB16], to create the convex optimization problems, together with the solver ECOS 2.0.7 DCB13. The symbolic computations were done in SYMPY 1.3 [JvMG12.

Investigated Data The experiment was carried out on a database containing 2020 randomly generated polynomials. The possible numbers of variables are $n=2,3,4,8,10$; the degree takes values $d=6,8,10,18,20,26,28$ and the number of terms can be $t=$ $6,9,12,20,24,30,50$. For each combinations we create instances, where the number of negative terms is one of a few fixed ratios of $t$. In particular, the size of (SAGE) grows quadratically in $t$. We created the database using POEM, and it is available in full at the homepage cited above. Our instances are a subset of those from [SdW18a. In that paper, we also describe their creation in more detail. The overall running time for all our instances was 6780.0 seconds.

Hardware and System We used an Intel Core i7-8550U CPU with $1.8 \mathrm{GHz}, 4$ cores, 8 threads and 16 GB of RAM under Ubuntu 18.04 for our computations.

Stopping Criteria For the accuracy of the solver and the precision of the rounding in PYTHON we used a tolerance of $\varepsilon=2^{-23}$. The restriction $t \leq 50$ was chosen, since otherwise we already encounter problem in the numerical solution of (SAGE). The bound $d<30$ was chosen, because for large degree we had a significant increase in the memory required to perform the rounding. Both thresholds were obtained experimentally.

5.2. Evaluation of the Experiment. In this section we present and evaluate the results of our experiment and highlight our most important findings, when investigating the computational data. We focus on the results given by the procedure optsage Algorithm 3.2 via SAGE decompositions and in the end give a comparison to optsonc. 


\begin{tabular}{|c|c|c|c|c|c|}
\hline \multicolumn{3}{|c|}{$d=8, t=20$} & \multicolumn{3}{|c|}{$d=10, t=24$} \\
\hline$n$ & bit size & time & $n$ & bit size & time \\
\hline 2 & 27723 & 5.82 & 2 & 38872 & 8.26 \\
\hline 3 & 23572 & 4.99 & 3 & 33938 & 7.26 \\
\hline 4 & 22965 & 4.83 & 4 & 31278 & 6.34 \\
\hline 8 & 5678 & 1.12 & 8 & 7042 & 1.42 \\
\hline 10 & 1749 & 0.35 & 10 & 3778 & 0.91 \\
\hline
\end{tabular}

TABLE 1. Dependency of the average bit size and the average running time of optsage, with the number of variables, for fixed values of degree $d$ and number of terms $t$; For $n=8$ we observe a drastic drop both in running time and bit size.

Running time decreases with growing number of variables. The formulation of (SAGE) shows that the size of the problem only depends on the number of terms $t$, but in the SAGE decomposition, the number of summands is the number of monomial nonsquares. Most significantly, for more variables, our generating algorithm simply results in a smaller number of these terms. Additionally, for $n \geq 8$ and $d \leq 10$, most exponents lie on faces of the Newton polytope. This leads to a simpler combinatorial structure, which we believe to result in lower bit sizes and thus in faster solving faster the exact LP from line 6 of optsonc. Next, we have more equality constraints in this LP, which could also improve the running time. Lastly, the exponential upper bound is just the worst case, which does not seem to actually happen among our examples. For some selected parameters, we exhibit that behavior in Table 1.

Dependency of bit size and running time of degree and terms To illustrate how bit size and running time of optsage vary for different degrees and numbers of terms, we restrict ourselves to at most 4 variables. Our numbers from the previous point show, that in these cases bit size and time are similar for fixed $(d, t)$, hence we may aggregate those instances. The results are shown in Table 2. We can see that running time and bit size roughly have a linear dependency. On the one hand, their growth is quadratic in the number of terms, which matches with the growth of the problem size in (SAGE). On the other hand, bit size and running time are basically unaffected by the degree. This shows that the bound, given in the worst case analysis, usually is not met.

Quality of the rounding-projection Our experiments verify that in the majority of cases the symbolical lower bound does not diverge far from the numerical bound. The detailed distribution is shown in Figure 1. Most notably, in 30 instances, the exact lower bound is even better than the numerical bound. In $81.9 \%$ of the instances, the exact bound differs by at most 0.001 from the numerical value. Only in 256 instances the difference lies above 1 . Thus, in the clear majority of examples, the lower bound in exact arithmetic does not differ much from the numerical bound. Also, among the instances with large difference, it can also be that the numerical solution actually lies far away from an exact solution. So it is unclear, whether a large difference is due to bad behavior of the numerical solution, or a large error in the rounding algorithm. 


\begin{tabular}{c|ccccccc}
$t \backslash d$ & 6 & 8 & 10 & 18 & 20 & 26 & 28 \\
\hline \multirow{2}{*}{6} & 912 & 1000 & 1002 & 1170 & 1014 & 955 & 900 \\
& 0.24 & 0.26 & 0.26 & 0.28 & 0.26 & 0.28 & 0.26 \\
\hline \multirow{2}{*}{9} & 2731 & 2673 & 2808 & 2890 & 2621 & 3166 & 2471 \\
& 0.66 & 0.65 & 0.70 & 0.68 & 0.61 & 0.82 & 0.62 \\
\hline \multirow{2}{*}{12} & 5599 & 6054 & 5449 & 5747 & 5478 & 6007 & 5027 \\
& 1.30 & 1.40 & 1.27 & 1.27 & 1.21 & 1.53 & 1.18 \\
\hline \multirow{2}{*}{20} & 9990 & 24078 & 20985 & 21364 & 19324 & 24096 & 17210 \\
& 2.26 & 5.08 & 4.40 & 4.43 & 3.96 & 5.23 & 3.59 \\
\hline \multirow{2}{*}{24} & $\times$ & 36301 & 33414 & 37080 & 29266 & 37618 & 28090 \\
& & 7.62 & 6.99 & 7.49 & 5.87 & 7.87 & 5.43 \\
\hline \multirow{2}{*}{30} & $\times$ & 57744 & 56354 & 61564 & 48622 & 59975 & 55000 \\
& & 11.90 & 11.44 & 12.57 & 9.32 & 12.76 & 10.80 \\
\hline \multirow{2}{*}{50} & $\times$ & $\times$ & 180971 & 174464 & 146218 & 196511 & 183598 \\
& & & 36.11 & 34.64 & 27.80 & 38.19 & 38.36 \\
\hline
\end{tabular}

TABLE 2. Bit size (upper part) and running time (lower part) of opt sage in dependency of the degree $d$ and the number of terms $t$ for up to 4 variables; A " $x$ " indicates, that we do not have instances with these parameters in our data set.

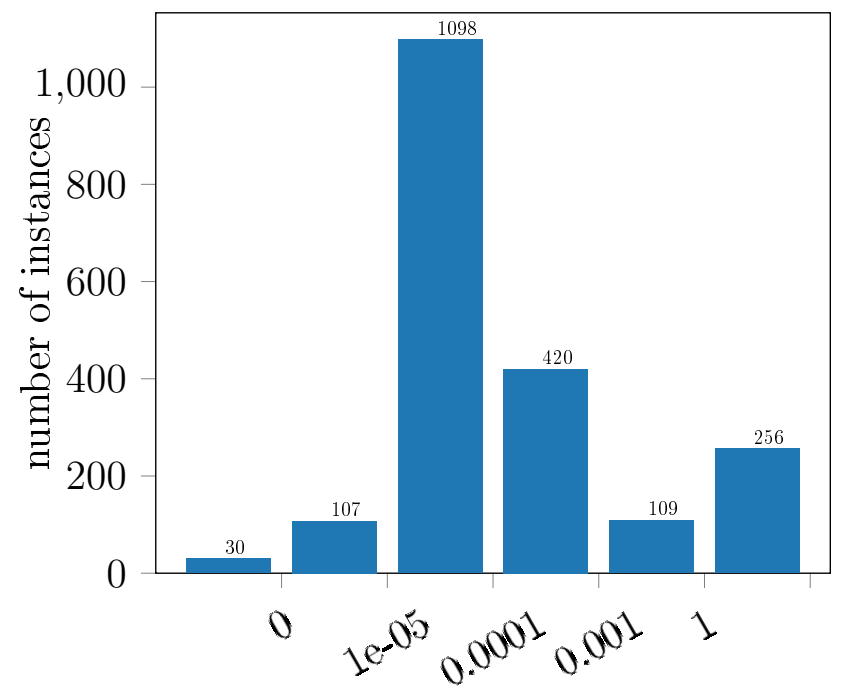

FIGURE 1. Number of instances where the difference of numerical lower bound and exact lower bound lies in the given interval; note that the exact bound sometimes is better.

Rounding time versus solving time In nearly every case the rounding procedure takes longer than the numerical solving. Only in 8 instances, the rounding took less time. The ratio of the rounding time to the total time ranges from $21.6 \%$ to $96.8 \%$, with an average of $88.6 \%$. However, one can implement the rounding procedure much closer to 


\begin{tabular}{r|rr|rr}
$t$ & bit size SONC & bit size SAGE & time SONC & time SAGE \\
\hline 6 & 432 & 1005 & 0.06 & 0.26 \\
9 & 806 & 2696 & 0.19 & 0.66 \\
12 & 1261 & 5568 & 0.37 & 1.29 \\
20 & 2592 & 19203 & 0.64 & 4.00 \\
24 & 3826 & 32543 & 0.97 & 6.66 \\
30 & 5029 & 53160 & 1.34 & 10.58 \\
50 & 10622 & 167971 & 3.95 & 32.78
\end{tabular}

TABLE 3. Comparison of running time and bit size of the certificates between optsonc and optsage; optsonc runs faster and has significantly smaller certificates than optsage.

the hardware level, instead of working in Python. Thus, we expect that these ratios can be significantly improved.

Comparison between SONC and SAGE In their qualitative behavior, optsonc and optsage are similar. However, optsonc runs faster and has smaller certificates than optsage, as shown in Table 3. But one should note that optsonc only computes some lower bound (not necessarily the optimal SONC-bound), whereas optsage computes the best bound, that can be obtained via this approach. Still it shows, that for very large instances, SONC is the method of choice, when other approaches fail due to the problem size.

Comparison with SOS For polynomials lying in the interior of the SOS cone from [MSED18, Table 2], we performed preliminary experiments with optsage and optsage, which are currently unable to provide nonnegativity certificates. For benchmarks from our database with $n \geq 8$ and $d \geq 10$, RealCertify often fails to provide SOS certificates. We plan to provide detailed experimental comparisons with SOS methods in the future.

\section{Conclusion And Outlook}

We make two main contributions in this paper. First, we present an algorithm to decide whether a given multivariate polynomial over the rationals lies in the interior of the SAGE cone. If that is the case, then the algorithm also computes a certificate in exact arithmetic. Additionally, we analyze the arithmetic complexity of the algorithm, which is polynomial in the degree and the number of terms, and exponential in the number of variables. Second, we use our numerical methods to obtain lower bounds via SAGE and apply a single iteration of the rounding-projection method, to obtain an exactly certified lower bound. This method, we run on a large number of test cases. Based on these experiments, we draw the following conclusions.

(1) In the majority of cases, the exact solution lies close to the numerical solution, with a difference of at most 0.001. For few instances, the exact lower bound is even better than the numerical one. 
(2) The running time and the bit size grow quadratically in the number of terms, which corresponds to the growth of the problem size.

(3) For the investigated parameters, increasing the degree or the number of variables does not increase the running time or the bit size. This also corresponds to the fact, that the size of the REP is independent of the degree and the number of variables.

(4) For very large instances, SONC should be the first choice, to obtain a certified bound, since it runs significantly faster than the other methods.

For future work, the most interesting development would be to have an REP-solver with arbitrary precision, so that we can actually implement intsage and compare it to similar approaches. Furthermore, in a significant amount of instances, we encountered computational problem, when calling optsonc or optsage. So we would like to increase the robustness of our implementation. Another issue, we have left out so far, is the presence of exponents of monomial non-squares, which lie on a face of the Newton polytope, that does not include the origin. These result in values $\lambda_{1, j}=0(\mathrm{SONC})$ or $\nu_{1}^{(j)}=0$ (SAGE), so our computation is undefined. However, these problems can be circumvented and we plan to do so in a future version of the software. Next, we plan to extend our framework to constrained problems and provide more detailed experimental comparisons with SOS-based approaches from [PP08, MSED18], as well as with methods based on critical points and cylindrical algebraic decomposition. Given a polynomial in the interior of the SAGE cone, our decision algorithm intsage is linear with respect to the distance of this polynomial to the border of the cone. In order to improve this bound, one could refine the bit size analysis for this distance. A further theoretical aim would be to analyze the boolean running time of intsage, which requires to prove bit complexity estimates for relative entropy optimization problems.

\section{REFERENCES}

[BCSS12] L. Blum, F. Cucker, M. Shub, and S. Smale. Complexity and real computation. Springer Science \& Business Media, 2012.

[BGHP05] B. Bank, M. Giusti, J. Heintz, and L.-M. Pardo. Generalized polar varieties: geometry and algorithms. Journal of complexity, 21(4):377-412, 2005.

[BKVH07] S. Boyd, S. Kim, L. Vandenberghe, and A. Hassibi. A tutorial on geometric programming. Optim. Eng., 8(1):67-127, 2007.

[BPR98] S. Basu, R. Pollack, and M.-F. Roy. A new algorithm to find a point in every cell defined by a family of polynomials. In Quantifier elimination and cylindrical algebraic decomposition. Springer-Verlag, 1998.

[BV04] S. Boyd and L. Vandenberghe. Convex optimization. Cambridge University Press, Cambridge, 2004.

[CHJL11] S. Chevillard, J. Harrison, M. Joldes, and C. Lauter. Efficient and accurate computation of upper bounds of approximation errors. Theoretical Computer Science, 412(16):1523 - 1543, 2011.

[Col75] G. E. Collins. Quantifier elimination for real closed fields by cylindrical algebraic decompostion. In ATFL 2nd GI Conf. Kaiserslautern, pages 134-183, 1975.

[CS16] V. Chandrasekaran and P. Shah. Relative Entropy Relaxations for Signomial Optimization. SIAM J. Optim., 26(2):1147-1173, 2016. 
[DB16] S. Diamond and S. Boyd. CVXPY: A Python-embedded modeling language for convex optimization. Journal of Machine Learning Research, 2016.

[DCB13] A. Domahidi, E. Chu, and S. Boyd. ECOS: An SOCP solver for embedded systems. In European Control Conference (ECC), pages 3071-3076, 2013.

[DIdW16] M. Dressler, S. Iliman, and T. de Wolff. An approach to constrained polynomial optimization via nonnegative circuit polynomials and geometric programming, 2016. To appear in the Journal of Symbolic Computation (MEGA 2017 special issue); see also arXiv:1602.06180.

[DIdW17] M. Dressler, S. Iliman, and T. de Wolff. A Positivstellensatz for Sums of Nonnegative Circuit Polynomials. SIAM J. Appl. Algebra Geom., 1(1):536-555, 2017.

[DPZ67] R. Duffin, E. Peterson, and C. Zener. Geometric programming: Theory and application. John Wiley \& Sons, Inc., New York-London-Sydney, 1967.

[dW15] T. de Wolff. Amoebas, nonnegative polynomials and sums of squares supported on circuits. Oberwolfach Rep., (23):1308-1311, 2015.

[GL96] G. H. Golub and C. F. V. Loan. Matrix Computations (3rd Ed.). Johns Hopkins University Press, Baltimore, MD, USA, 1996.

[GM12] M. Ghasemi and M. Marshall. Lower bounds for polynomials using geometric programming. SIAM J. Optim., 22(2):460-473, 2012.

[GM13] M. Ghasemi and M. Marshall. Lower bounds for a polynomial on a basic closed semialgebraic set using geometric programming, 2013. Preprint, arxiv:1311.3726.

[GV88] D. Grigoriev and N. Vorobjov. Solving systems of polynomials inequalities in subexponential time. Journal of Symbolic Computation, 5:37-64, 1988.

$\left[\mathrm{HAB}^{+} 17\right]$ T. Hales, M. Adams, G. Bauer, D. T. Dat, J. Harrison, H. L. Truong, C. Kaliszyk, V. Magron, S. Mclaughlin, N. T. Thang, N. Q. Truong, T. Nipkow, S. Obua, J. Pleso, J. Rute, A. Solovyev, T. T. H. An, T. N. Trung, T. T. Diep, J. Urban, V. K. Ky, and R. Zumkeller. A Formal Proof of the Kepler Conjecture. Forum of Mathematics, Pi, 5, 2017.

[IdW16] S. Iliman and T. de Wolff. Amoebas, nonnegative polynomials and sums of squares supported on circuits. Res. Math. Sci., 3:3:9, 2016.

[JvMG12] D. Joyner, O. Čertík, A. Meurer, and B. E. Granger. Open source computer algebra systems: Sympy. ACM Commun. Comput. Algebra, 45(3/4):225-234, January 2012.

[Las01] J.-B. Lasserre. Global optimization with polynomials and the problem of moments. SIAM Journal on Optimization, 11(3):796-817, 2001.

[Las06] J.-B. Lasserre. Convergent SDP-Relaxations in Polynomial Optimization with Sparsity. SIAM Journal on Optimization, 17(3):822-843, 2006.

[Las10] J.-B. Lasserre. Moments, positive polynomials and their applications, volume 1 of Imperial College Press Optimization Series. Imperial College Press, London, 2010.

[Lau09] M. Laurent. Sums of squares, moment matrices and optimization over polynomials. In Emerging applications of algebraic geometry, volume 149 of IMA Vol. Math. Appl., pages 157-270. Springer, New York, 2009.

[LTY17] J.-B. Lasserre, K.-C. Toh, and S. Yang. A bounded degree SOS hierarchy for polynomial optimization. EURO Journal on Computational Optimization, 5(1-2):87-117, 2017.

[MAGW15] V. Magron, X. Allamigeon, S. Gaubert, and B. Werner. Formal proofs for Nonlinear Optimization. Journal of Formalized Reasoning, 8(1):1-24, 2015.

[MCD17] V. Magron, G. Constantinides, and A. Donaldson. Certified Roundoff Error Bounds Using Semidefinite Programming. ACM Trans. Math. Softw., 43(4):1-34, 2017.

[MCW18] R. Murray, V. Chandrasekaran, and A. Wierman. Newton polytopes and relative entropy optimization. arXiv preprint arXiv:1810.01614, 2018.

[MD18a] V. Magron and M. S. E. Din. On Exact Polya and Putinar's Representations. In ISSAC'18: Proceedings of the 2018 ACM International Symposium on Symbolic and Algebraic Computation. ACM, New York, NY, USA, 2018. 
[MD18b] V. Magron and M. S. E. Din. RealCertify: a Maple package for certifying non-negativity. In ISSAC'18: Proceedings of the 2018 ACM International Symposium on Symbolic and Algebraic Computation. ACM, New York, NY, USA, 2018.

[MDS18] V. Magron, M. S. E. Din, and M. Schweighofer. Algorithms for weighted sum of squares decomposition of non-negative univariate polynomials. Journal of Symbolic Computation, 2018.

[Mig92] M. Mignotte. Mathematics for Computer Algebra. Springer-Verlag New York, Inc., New York, NY, USA, 1992.

[MSED18] V. Magron and M. Safey El Din. On Exact Polya, Hilbert-Artin and Putinar's Representations. arXiv preprint arXiv:1811.10062, 2018.

[Oxl11] J. Oxley. Matroid theory, volume 21 of Oxford Graduate Texts in Mathematics. Oxford University Press, Oxford, second edition, 2011.

[Par00] P. A. Parrilo. Structured Semidefinite Programs and Semialgebraic Geometry Methods in Robustness and Optimization. PhD thesis, California Inst. Tech., 2000.

[PP08] H. Peyrl and P. Parrilo. Computing sum of squares decompositions with rational coefficients. Theoretical Computer Science, 409(2):269-281, 2008.

[Ren88] J. Renegar. A faster PSPACE algorithm for deciding the existential theory of the reals. In Foundations of Computer Science, 1988., 29th Annual Symposium on, pages 291-295. IEEE, 1988.

[Ren01] J. Renegar. A Mathematical View of Interior-Point Methods in Convex Optimization. SIAM, 2001.

[RTAL13] C. Riener, T. Theobald, L. J. Andrén, and J.-B. Lasserre. Exploiting Symmetries in SDPRelaxations for Polynomial Optimization. Mathematics of Operations Research, 38(1):122141, 2013, http://dx.doi.org/10.1287/moor.1120.0558.

[SdW18a] H. Seidler and T. de Wolff. An experimental comparison of sonc and sos certificates for unconstrained optimization. arXiv preprint arXiv:1808.08431, 2018.

[SdW18b] H. Seidler and T. de Wolff. POEM: Effective methods in polynomial optimization, version 0.1.1.0(a). https://www3.math.tu-berlin.de/combi/RAAGConOpt/poem.html, April 2018.

[WKKM06] H. Waki, S. Kim, M. Kojima, and M. Muramatsu. Sums of Squares and Semidefinite Programming Relaxations for Polynomial Optimization Problems with Structured Sparsity. SIAM Journal on Optimization, 17(1):218-242, 2006.

[WLT18] T. Weisser, J.-B. Lasserre, and K.-C. Toh. Sparse-BSOS: a bounded degree SOS hierarchy for large scale polynomial optimization with sparsity. Mathematical Programming Computation, 10(1):1-32, 2018. 


\section{Appendix A. Appendix}

A.1. Proof of Lemma 4.4. We start with the first claim. By Lemma 4.2, there exist $\widetilde{\boldsymbol{\nu}}, \widetilde{\boldsymbol{c}}$ and $\varepsilon \in \mathbb{Q}_{>0}$, with $\tau(\varepsilon) \in \mathcal{O}\left(\tau \cdot(4 d+6)^{3 n+3}\right)$, such that $(\widetilde{\boldsymbol{\nu}}, \widetilde{\boldsymbol{c}})$ satisfies $\sum_{j=1}^{t} \boldsymbol{c}^{(j)}=\boldsymbol{b}$, $\widetilde{\boldsymbol{c}}_{\backslash j}^{(j)}, \widetilde{\boldsymbol{\nu}}_{\backslash j}^{(j)}>\mathbf{0}, D\left(\widetilde{\boldsymbol{\nu}}_{\backslash j}^{(j)}, e \widetilde{\boldsymbol{c}}_{\backslash j}^{(j)}\right)+\varepsilon \leq \widetilde{c}_{j}^{(j)}$, for all $j=1, \ldots, t$. Let us define $\delta:=\frac{\varepsilon}{2(t-1)}$ and $\boldsymbol{c}$ such that $c_{i}^{(j)}:=\widetilde{c}_{i}^{(j)}+\frac{\varepsilon}{2}$ for all $i \neq j=1, \ldots, t$, and $c_{j}^{(j)}:=\widetilde{c}_{j}^{(j)}-t \delta$, for $j=1, \ldots, t$. Thus, one has $\sum_{j=1}^{t} \boldsymbol{c}^{(j)}=\boldsymbol{b}$. For all $j=1, \ldots, t$, one has $\boldsymbol{\nu}_{{ }_{j}}^{(j)}>\mathbf{0}$. Combining this together with the fact that the $\log$ function is increasing yields

$$
D\left(\boldsymbol{\nu}_{\backslash j}^{(j)}, e\left(\boldsymbol{c}_{\backslash j}^{(j)}+\delta \mathbf{1}\right)\right)+\frac{\varepsilon}{2} \leq D\left(\boldsymbol{\nu}_{\backslash j}^{(j)}, e \boldsymbol{c}_{\backslash j}^{(j)}\right) \leq \widetilde{c}_{j}^{(j)}-\frac{\varepsilon}{2}=c_{j}^{(j)} .
$$

Using that for all $d \geq 2$,

$$
\begin{aligned}
t & \leq\left(\begin{array}{c}
n+d \\
n
\end{array}\right)=\frac{(n+d) \cdots(d+1)}{n !}=\left(1+\frac{d}{n}\right)\left(1+\frac{d}{n-1}\right) \cdots(1+d) \\
& \leq d^{n-1}(1+d) \leq 2 d^{n},
\end{aligned}
$$

one has $\tau(t) \in \mathcal{O}\left(n \log _{2}(d)\right)$. Since $\tau(\delta) \leq \tau(\varepsilon)+\tau(t)$, we obtain the first claim.

To prove the second claim, we rely on the following three auxiliary inequalities.

For $\nu, \delta$ such that $0<\nu<\delta \leq \frac{1}{2}$, one has

$$
(\nu+\delta) \log (\nu+\delta) \leq 0,
$$

since $0<\nu+\delta<1$ and the function $x \mapsto x \log x$ is negative on $(0,1)$.

For $\nu, \delta$ such that $0<\delta \leq \nu$, one has

$$
(\nu+\delta) \log (1+\delta / \nu) \leq 2 \delta,
$$

since $\nu+\delta \leq 2 \nu$ and $\log (1+\delta / \nu) \leq \delta / \nu$.

For each $\nu, \delta, c>0$ such that $\nu \geq 1$, one has

$$
\delta \log \left(\frac{\nu}{e c}\right) \leq \delta \max \left\{0, \nu \log \left(\frac{\nu}{e c}\right)\right\} .
$$

Indeed, if $\nu \leq e c$, the left hand side is less than 0 . Otherwise, $\nu \geq 1$ implies that $\log \left(\frac{\nu}{e c}\right) \leq \nu \log \left(\frac{\nu}{e c}\right)$.

Now, by the first claim, there exist $\varepsilon_{\boldsymbol{c}}, \varepsilon \in \mathbb{Q}_{>0}$, with $\tau\left(\varepsilon_{\boldsymbol{c}}\right), \tau(\varepsilon) \in \mathcal{O}\left(\tau \cdot(4 d+6)^{3 n+3}\right)$, and $\widetilde{\boldsymbol{\nu}}, \widetilde{\boldsymbol{c}}$ satisfying $\widetilde{\boldsymbol{\nu}}_{{ }_{j}}^{(j)}>\mathbf{0}, \widetilde{\boldsymbol{c}}_{\backslash j}^{(j)}>\varepsilon_{\boldsymbol{c}} \mathbf{1}$, and $D\left(\widetilde{\boldsymbol{\nu}}_{\backslash j}^{(j)}, e \widetilde{\boldsymbol{c}}_{{ }_{j}}^{(j)}\right)+\varepsilon \leq \widetilde{c}_{j}^{(j)}$, for all $j=1, \ldots, t$. For each $\delta \in \mathbb{Q}_{>0}$ with $\delta \leq \frac{1}{2}$, and all $j=1, \ldots, t$, one has:

$$
D\left(\widetilde{\boldsymbol{\nu}}_{\backslash j}^{(j)}+\delta \mathbf{1}, e \widetilde{\boldsymbol{c}}_{\backslash j}^{(j)}\right)=\sum_{i \neq j}\left(\widetilde{\nu}_{i}^{(j)}+\delta\right) \log \frac{\widetilde{\nu}_{i}^{(j)}+\delta}{e \widetilde{c}_{i}^{(j)}} .
$$

We give an upper bound of each summand of the right hande side, depending on the value of $\widetilde{\nu}_{i}^{j}$, for all $i, j=1, \ldots, t$ and $i \neq j$. Note that $-\log \left(e c_{i}^{j}\right) \leq \log \left(e \varepsilon_{c}\right)$, for all $i, j=1, \ldots, t$ and $i \neq j$. 
If $0<\widetilde{\nu}_{i}^{j} \leq \delta \leq \frac{1}{2}$, one has by $(\mathrm{A} .1)$

$$
\begin{aligned}
\left(\widetilde{\nu}_{i}^{(j)}+\delta\right) \log \frac{\widetilde{\nu}_{i}^{(j)}+\delta}{e \widetilde{c}_{i}^{(j)}} & \leq\left(\widetilde{\nu}_{i}^{(j)}+\delta\right) \log \left(\widetilde{\nu}_{i}^{(j)}+\delta\right)-\left(\widetilde{\nu}_{i}^{(j)}+\delta\right) \log \left(e \widetilde{c}_{i}^{(j)}\right) \\
& \leq 2 \delta \log \left(e \varepsilon_{\boldsymbol{c}}\right) .
\end{aligned}
$$

If $\delta \leq \widetilde{\nu}_{i}^{j} \leq 1$, one has

$$
\begin{aligned}
\left(\widetilde{\nu}_{i}^{(j)}+\delta\right) & \log \frac{\widetilde{\nu}_{i}^{(j)}+\delta}{e \widetilde{c}_{i}^{(j)}} \\
& \leq \widetilde{\nu}_{i}^{(j)} \log \frac{\widetilde{\nu}_{i}^{(j)}}{e \widetilde{c}_{i}^{(j)}}+\delta \log \frac{\widetilde{\nu}_{i}^{(j)}}{e \widetilde{c}_{i}^{(j)}}+\left(\widetilde{\nu}_{i}^{(j)}+\delta\right) \log \left(1+\frac{\delta}{\widetilde{\nu}_{i}^{(j)}}\right) \\
& \leq \widetilde{\nu}_{i}^{(j)} \log \frac{\widetilde{\nu}_{i}^{(j)}}{e \widetilde{c}_{i}^{(j)}}+2 \delta \log \left(e \varepsilon_{\boldsymbol{c}}\right)+2 \delta
\end{aligned}
$$

where we use the fact that $\delta \log \widetilde{\nu}_{i}^{(j)} \leq 0$ and bound the last term of the right hand side via (A.2).

If $\widetilde{\nu}_{i}^{J} \geq 1$, we write the first inequality as in the former case and obtain

$$
\left(\widetilde{\nu}_{i}^{(j)}+\delta\right) \log \frac{\widetilde{\nu}_{i}^{(j)}+\delta}{e \widetilde{c}_{i}^{(j)}} \leq \widetilde{\nu}_{i}^{(j)} \log \frac{\widetilde{\nu}_{i}^{(j)}}{e \widetilde{c}_{i}^{(j)}}+\delta \max \left\{0, \widetilde{\nu}_{i}^{(j)} \log \frac{\widetilde{\nu}_{i}^{(j)}}{e \widetilde{c}_{i}^{(j)}}\right\}+2 \delta,
$$

where we rely on (A.3) to bound the second term and the fact that $\delta+\widetilde{\nu}_{i}^{(j)} \leq 2 \widetilde{\nu}_{i}^{(j)}$ together with $\log \left(1+\frac{\delta}{\widetilde{\nu}_{i}^{(j)}}\right) \leq \frac{\delta}{\widetilde{\nu}_{i}^{(j)}}$ to bound the last term.

In the worst case, we obtain

$$
\begin{aligned}
D\left(\widetilde{\boldsymbol{\nu}}_{\backslash j}^{(j)}+\delta \mathbf{1}, e \widetilde{\boldsymbol{c}}_{\backslash j}^{(j)}\right) & \left.\leq(1+2 \delta) D\left(\widetilde{\boldsymbol{\nu}}_{\backslash j}^{(j)}, e \widetilde{\boldsymbol{c}}_{\backslash j}^{(j)}\right)+2 \delta(t-1)\left|\log \left(e \varepsilon_{\boldsymbol{c}}\right)\right|+1\right) \\
& \leq \widetilde{c}_{j}^{(j)}+2 \delta \widetilde{c}_{j}^{(j)}-(1+2 \delta) \varepsilon+2 \delta t\left|\log \left(e \varepsilon_{\boldsymbol{c}}\right)\right| \\
& \leq \widetilde{c}_{j}^{(j)}-(1+2 \delta) \varepsilon+2 \delta\left(\left|b_{j}\right|+t\left|\log \left(e \varepsilon_{\boldsymbol{c}}\right)\right|\right),
\end{aligned}
$$

using $\widetilde{c}_{j}^{(j)}=b_{j}-\mathbf{1} \cdot \widetilde{c}_{\backslash j}^{(j)} \leq b_{j}$, thus $\widetilde{c}_{j}^{(j)} \leq \max \left\{0, b_{j}\right\} \leq\left|b_{j}\right|$.

To ensure that $D\left(\widetilde{\boldsymbol{\nu}}_{\backslash j}^{(j)}+\delta \mathbf{1}, e \widetilde{\boldsymbol{c}}_{\backslash j}^{(j)}\right)+\frac{\varepsilon}{2} \leq \widetilde{c}_{j}^{(j)}$, it is sufficient to have $-(1+4 \delta) \varepsilon+$ $4 \delta\left(\left|b_{j}\right|+t\left|\log \left(e \varepsilon_{c}\right)\right|\right) \leq 0$, which is guaranteed by selecting the largest positive rational $\delta$ such that $\delta \leq \frac{\varepsilon}{4\left(\left|b_{j}\right|+t\left|\log \left(e \varepsilon_{c}\right)\right|-\varepsilon\right)}$. Since $\tau\left(\left|b_{j}\right|\right) \leq \tau, \tau(t) \in \mathcal{O}\left(d \log _{2} n\right)$, $\left.\left|\log \left(e \varepsilon_{\boldsymbol{c}}\right)\right|\right) \leq \tau\left(\varepsilon_{\boldsymbol{c}}\right)$, and $\tau\left(\varepsilon_{\boldsymbol{c}}\right), \tau(\varepsilon) \in \mathcal{O}\left(\tau \cdot(4 d+6)^{3 n+3}\right)$, one can select $\delta$ with bit size at most $\mathcal{O}\left(\tau \cdot(4 d+6)^{3 n+3}\right)$.

The proof of the third claim is very similar and we omit it for the sake of conciseness. 


\section{A.2. An Example of Exact SAGE Decomposition. Let}

$$
\begin{aligned}
f(\boldsymbol{x})= & 277-1 x_{2}^{2}+159 x_{2}^{2} x_{3}^{6}+275 x_{2}^{4}-112 x_{1}^{1} x_{2}^{1} x_{3}^{2}+23 x_{1}^{1} x_{2}^{2} x_{3}^{3}+338 x_{1}^{2} x_{3}^{4}+166 x_{1}^{2} x_{2}^{1} x_{3}^{1} \\
& -89 x_{1}^{2} x_{2}^{1} x_{3}^{2}-19 x_{1}^{2} x_{2}^{2} x_{3}^{1}+74 x_{1}^{2} x_{2}^{2} x_{3}^{2}+268 x_{1}^{6} x_{3}^{2} .
\end{aligned}
$$

Our optimization algorithm optsage returns $(\boldsymbol{\nu}, \boldsymbol{c})$ corresponding to the following exact rational SAGE decomposition: $f(\boldsymbol{x})=\sum_{j=1}^{12} f_{j}(\boldsymbol{x})$, where $f_{j}$ is the polynomial with coefficient vector $\boldsymbol{c}^{(j)}$ for $j \in\{1, \ldots, 12\}, f_{j}=0$ for $j \in\{1,3,4,7,11,12\}$, and

$$
\begin{aligned}
& \boldsymbol{\nu}^{(2)}=\left(\frac{1494563}{131072},-\frac{1494563}{65536}, 0, \frac{1494563}{131072}, 0,0,0,0,0,0,0,0\right) \\
& f_{2}=\frac{7050}{5161}-\frac{47048170074075847768}{2063044386600414657} x_{2}^{2}+\frac{51364821929347737990}{176335433100237218699413} x_{2}^{2} x_{3}^{6}+\frac{1284035788022670567885}{13490375489389305583} x_{2}^{4}+ \\
& \frac{83062810507300624}{22200197261503152705103} x_{1}^{1} x_{2}^{1} x_{3}^{2}+\frac{16345503627618}{597428155608650885} x_{1}^{1} x_{2}^{2} x_{3}^{3}+\frac{151893109261090080}{12048313292258664398599} x_{1}^{2} x_{3}^{4}+ \\
& \frac{6887262866990276056}{3068799030848037634611567} x_{1}^{2} x_{2}^{1} x_{3}^{1}+\frac{584984347065145672}{102911615960676281785795} x_{1}^{2} x_{2}^{1} x_{3}^{2}+\frac{35180881401784}{5417152094682478713} x_{1}^{2} x_{2}^{2} x_{3}^{1}+ \\
& \frac{910066980468240}{55380305977850685287} x_{1}^{2} x_{2}^{2} x_{3}^{2}+\frac{7052856072901897195}{672038441901303429467107} x_{1}^{6} x_{3}^{2} \\
& \boldsymbol{\nu}^{(5)}=\left(\frac{2763713}{131072}, \frac{1404885}{65536}, \frac{40783}{131072}, \frac{2618913}{131072},-\frac{1033291}{8192}, \frac{46873}{65536}, \frac{8086635}{131072}, \frac{2825}{16384}, \frac{16731}{65536}, \frac{5689}{32768}, \frac{4177}{16384}, \frac{3431}{65536}\right) \\
& f_{5}=\frac{4895}{6532}+\frac{397583449413593513}{62516496563648929} x_{2}^{2}+\frac{4282065321967986445380570}{176335433100237218699413} x_{2}^{2} x_{3}^{6}+\frac{5340068537046034265265}{107923003915114444664} x_{2}^{4}- \\
& \frac{797551428730610634080}{6323041088437240873} x_{1}^{1} x_{2}^{1} x_{3}^{2}+\frac{624998409184656940}{119485631121730177} x_{1}^{1} x_{2}^{2} x_{3}^{3}+\frac{2505598243831220279021280}{12048313292258664398599} x_{1}^{2} x_{3}^{4}+ \\
& \frac{317731662232293486548800}{3068799030848037634611567} x_{1}^{2} x_{2}^{1} x_{3}^{1}+\frac{7986264919496237716952}{20582323192135256357159} x_{1}^{2} x_{2}^{1} x_{3}^{2}+\frac{4894573585100619175}{16251456284047436139} x_{1}^{2} x_{2}^{2} x_{3}^{1}+ \\
& \frac{62000736112963044488}{55380305977850685287} x_{1}^{2} x_{2}^{2} x_{3}^{2}+\frac{98753640749442855613080}{672038441901303429467107} x_{1}^{6} x_{3}^{2} \\
& \boldsymbol{\nu}^{(6)}=\left(\frac{24235}{65536}, \frac{11469}{16384}, \frac{731111}{65536}, \frac{413881}{65536}, \frac{75499}{65536},-\frac{2262417}{65536}, \frac{224607}{65536}, \frac{48937}{65536}, \frac{41999}{32768}, \frac{5895}{4096}, \frac{459131}{65536}, \frac{30411}{32768}\right) \\
& f_{6}=\frac{11}{6124}+\frac{175751812330814062}{6189133159801243971} x_{2}^{2}+\frac{20993411202657969962132460}{176335433100237218699413} x_{2}^{2} x_{3}^{6}+\frac{230789345356723546855}{107923003915114444664} x_{2}^{4}+ \\
& \frac{3497123471444427592320}{22200197261503152705103} x_{1}^{1} x_{2}^{1} x_{3}^{2}-\frac{20624391427747375598}{597428155608650885} x_{1}^{1} x_{2}^{2} x_{3}^{3}+\frac{19031996317336046221320}{12048313292258664398599} x_{1}^{2} x_{3}^{4}+ \\
& \frac{188140322704886960215808}{3068799030848037634611567} x_{1}^{2} x_{2}^{1} x_{3}^{1}+\frac{27412383525960721370432}{102911615960676281785795} x_{1}^{2} x_{2}^{1} x_{3}^{2}+\frac{616440189731061280}{1805717364894159571} x_{1}^{2} x_{2}^{2} x_{3}^{1}+ \\
& \frac{232955118417446202960}{55380305977850685287} x_{1}^{2} x_{2}^{2} x_{3}^{2}+\frac{239384185705575737209071}{672038441901303429467107} x_{1}^{6} x_{3}^{2} \\
& \boldsymbol{\nu}^{(8)}=\left(\frac{5736955}{131072}, \frac{1718597}{65536}, \frac{2677}{65536}, \frac{283517}{16384}, \frac{298687}{65536}, \frac{5897}{32768}, \frac{1205677}{131072},-\frac{1398845}{8192}, \frac{338517}{65536}, \frac{488327}{32768}, \frac{37365}{8192}, \frac{1469765}{32768}\right) \\
& f_{8}=\frac{16553}{6389}+\frac{80194631513313539404}{6189133159801243971} x_{2}^{2}+\frac{936097366115717531112852}{176335433100237218699413} x_{2}^{2} x_{3}^{6}+\frac{962838001240718755020}{13490375489389305583} x_{2}^{4}+ \\
& \frac{168516279078159280107840}{22200197261503152705103} x_{1}^{1} x_{2}^{1} x_{3}^{2}+\frac{261920871973041930}{119485631121730177} x_{1}^{1} x_{2}^{2} x_{3}^{3}+\frac{622190516331822168107520}{12048313292258664398599} x_{1}^{2} x_{3}^{4}- \\
& \frac{174673447091258679729208576}{1022933010282679211537189} x_{1}^{2} x_{2}^{1} x_{3}^{1}+\frac{1345600783114821896667952}{102911615960676281785795} x_{1}^{2} x_{2}^{1} x_{3}^{2}+\frac{233241950370990311440}{5417152094682478713} x_{1}^{2} x_{2}^{2} x_{3}^{1}+ \\
& \frac{1847380121666164588230}{55380305977850685287} x_{1}^{2} x_{2}^{2} x_{3}^{2}+\frac{140920372909036228167589650}{672038441901303429467107} x_{1}^{6} x_{3}^{2}
\end{aligned}
$$




$$
\begin{aligned}
\boldsymbol{\nu}^{(9)}= & \left(\frac{942879}{131072}, \frac{66451}{8192}, \frac{13687}{131072}, \frac{291521}{32768}, \frac{487245}{65536}, \frac{29821}{65536}, \frac{2150473}{65536}, \frac{252191}{32768},-\frac{3460417}{32768}, \frac{170395}{16384}, \frac{548667}{65536}, \frac{925733}{65536}\right) \\
f_{9}= & \frac{181}{1076}+\frac{3266564780864805056}{2063044386600414657} x_{2}^{2}+\frac{945568751423587451936415}{176335433100237218699413} x_{2}^{2} x_{3}^{6}+\frac{195552867021926712375}{13490375489389305583} x_{2}^{4}+ \\
& \frac{108598341793757685149280}{22200197261503152705103} x_{1}^{1} x_{2}^{1} x_{3}^{2}+\frac{261624143096996295}{119485631121730177} x_{1}^{1} x_{2}^{2} x_{3}^{3}+\frac{876811143650234639492646}{12048313292258664398599} x_{1}^{2} x_{3}^{4}+ \\
& \frac{9330362356036127696945600}{3068799030848037634611567} x_{1}^{2} x_{2}^{1} x_{3}^{1}-\frac{10867831413671203786608456}{102911615960676281785795} x_{1}^{2} x_{2}^{1} x_{3}^{2}+\frac{192909634965734635520}{16251456284047436139} x_{1}^{2} x_{2}^{2} x_{3}^{1}+ \\
& \frac{1339551331036614124520}{55380305977850685287} x_{1}^{2} x_{2}^{2} x_{3}^{2}+\frac{17532007073614874704137330}{672038441901303429467107} x_{1}^{6} x_{3}^{2} \\
\boldsymbol{\nu}^{(10)}= & \left.\frac{732091}{262144}, \frac{167933}{32768}, \frac{14541}{131072}, \frac{7773365}{262144}, \frac{1327}{512}, \frac{29683}{65536}, \frac{272641}{131072}, \frac{293905}{65536}, \frac{214965}{65536},-\frac{4886959}{65536}, \frac{144047}{32768}, \frac{1284635}{65536}\right) \\
f_{10}= & \frac{157}{2744}+\frac{5424537902241772912}{6189133159801243971} x_{2}^{2}+\frac{880139855950527034906380}{176335433100237218699413} x_{2}^{2} x_{3}^{6}+\frac{571069367996398273530}{13490375489389305583} x_{2}^{4}+ \\
& \frac{33169145578648933133280}{22200197261503152705103} x_{1}^{1} x_{2}^{1} x_{3}^{2}+\frac{228162076394260360}{119485631121730177} x_{1}^{1} x_{2}^{2} x_{3}^{3}+\frac{48697840759706172793616}{12048313292258664398599} x_{1}^{2} x_{3}^{4}+ \\
& \frac{1587820308255205569373114}{1022933010282679211537189} x_{1}^{2} x_{2}^{1} x_{3}^{1}+\frac{59150503389680767180777}{20582323192135256357159} x_{1}^{2} x_{2}^{1} x_{3}^{2}-\frac{403951932103643744176}{5417152094682478713} x_{1}^{2} x_{2}^{2} x_{3}^{1}+ \\
& \frac{616254425060782282800}{55380305977850685287} x_{1}^{2} x_{2}^{2} x_{3}^{2}+\frac{21315777567587124730738350}{672038441901303429467107} x_{1}^{6} x_{3}^{2}
\end{aligned}
$$

Victor Magron, CNRS laAs, 7 avenue du Colonel Roche, F-31031 Toulouse Cédex 4 , FRANCE

E-mail address: victor.magron@laas.fr

Henning Seidler, Technische Universität Berlin, Institut für Mathematik, Strasse des 17. Juni 136, 10623 Berlin, Germany

E-mail address: seidler@math.tu-berlin.de

Timo de Wolff, Technische Universität Berlin, Institut für Mathematik, Strasse des 17. Juni 136, 10623 Berlin, Germany

E-mail address: dewolff@math.tu-berlin.de 